\title{
Spiral order from orientationally correlated random bonds in classical $X Y$ models
}

\author{
Andrea Scaramucci, ${ }_{1}^{1}$ Hiroshi Shinaoka $\odot,{ }^{2,3,4}$ Maxim V. Mostovoy, ${ }^{5}$ Rui Lin $\odot,{ }^{2}$ Christopher Mudry, ${ }^{6,7}$ and Markus Müller $\odot^{6,8}$ \\ ${ }^{1}$ Laboratory for Scientific Development and Novel Materials, Paul Scherrer Institut, 5235 Villigen PSI, Switzerland \\ ${ }^{2}$ Institute for Theoretical Physics, ETH Zürich, 8093 Zürich, Switzerland \\ ${ }^{3}$ Department of Physics, University of Fribourg, 1700 Fribourg, Switzerland \\ ${ }^{4}$ Department of Physics, Saitama University, 338-8570 Saitama, Japan \\ ${ }^{5}$ Zernike Institute for Advanced Materials, University of Groningen, Nijenborgh 4, 9747 AG Groningen, Netherlands \\ ${ }^{6}$ Condensed Matter Theory Group, Paul Scherrer Institute, 5232 Villigen PSI, Switzerland \\ ${ }^{7}$ Institute of Physics, École Polytechnique Fédérale de Lausanne, 1015 Lausanne, Switzerland \\ ${ }^{8}$ Abdus Salam International Centre for Theoretical Physics, 34151 Trieste, Italy
}

(Received 18 December 2019; accepted 4 February 2020; published 12 March 2020)

\begin{abstract}
We discuss the stability of ferromagnetic long-range order in three-dimensional classical $X Y$ ferromagnets upon substitution of a small subset of equally oriented bonds by impurity bonds, on which the ferromagnetic exchange $J_{\perp}>0$ is replaced by a strong antiferromagnetic coupling $J_{\text {imp }}<0$. In the impurity-free limit, the effective low-energy Hamiltonian is that of spin waves. In the presence of a single, sufficiently strongly frustrating impurity bond, the ground state is twofold degenerate, corresponding to either clockwise or counterclockwise canting of the spins in the vicinity of the impurity bond. For a small but finite concentration of impurity bonds, the effective low-energy Hamiltonian is that of Ising variables encoding the sense of rotation of the local canting around the impurities. Those degrees of freedom interact pairwise through a dipolar interaction mediated by spin waves. A spatially random distribution of impurities leads to a ferromagnetic Ising ground state, which indicates the instability of the $X Y$ ferromagnet towards a spiral state, with wave vector and transition temperature both proportional to the concentration of impurity bonds. This mechanism of spiral order by disorder is relevant for magnetic materials such as $\mathrm{YBaCuFeO}_{5}$, for which our theory predicts a ratio between the spiral ordering temperature and the modulus of the spiral wave vector close to the measured ones.
\end{abstract}

DOI: 10.1103/PhysRevResearch.2.013273

\section{INTRODUCTION}

Insulating magnets supporting long-range magnetic spiral order are of technological interest as they can display magnetically induced ferroelectricity [1-4]. In prototypical spin-spiral multiferroics, e.g., $R \mathrm{MnO}_{3}\left(R=\mathrm{Tb}^{3+}, \mathrm{Dy}^{3+}\right.$, etc. $)$ $[5,6]$, a magnetic spiral phase can be stabilized by the competition between nearest-neighbor and further-neighbor magnetic exchange interactions with opposite signs [7,8]. However, the resulting frustration only induces spiral states if further-neighbor couplings are sufficiently strong as compared to nearest-neighbor couplings. The latter are typically much bigger in magnitude, except under special circumstances that lead to their suppression. In such exceptional cases, the characteristic exchange scale is set by the further-neighbor interactions and is thus very weak, entailing a low spiral ordering temperature.

In order to engineer magnetic insulators with magnetic spiral order established at high temperatures, it is of fundamental interest to investigate analogous mechanisms. An interesting

Published by the American Physical Society under the terms of the Creative Commons Attribution 4.0 International license. Further distribution of this work must maintain attribution to the author(s) and the published article's title, journal citation, and DOI. route was suggested by the study of Ivanov et al. [9], who considered a Heisenberg antiferromagnet on a square lattice, in which every other horizontal nearest-neighbor bond in a staggered pattern was replaced by a ferromagnetic coupling. Sufficiently strongly frustrating bonds were shown to induce a magnetic spiral order. From the experimental side, there are interesting hints that a similar mechanism might be tied to the presence of disorder. Indeed, certain insulating compounds containing some degree of chemical disorder were reported to stabilize magnetic spiral order [10-14] at high temperatures. For example, the transition temperatures to the magnetic spiral phase were found to range from 180 to $310 \mathrm{~K}$ [12,14-17] in $\mathrm{YBaCuFeO}_{5}$, whereby several further characteristics of the spiral depend on the degree of disorder. A recent experimental study in Ref. [18] demonstrated the same phenomenology for the family of layered perovskites $A B \mathrm{CuFeO}_{5}$, where $A$ stands for a rare-earth ion and $B$ stands for $\mathrm{Ba}$ or Sr. This empirical observation suggests the possibility that, for some materials, a magnetic spiral order might be induced by some impurity bonds formed by nearest-neighbor magnetic ions whose exchange coupling frustrates the order that would be established in their absence. Recent Monte Carlo simulations have confirmed this conjecture in a model describing the family of layered perovskites $A B \mathrm{CuFeO}_{5}$ with disorder in the spatial location of the magnetic $\mathrm{Cu}$ and Fe ions [19]. The latter was assumed to result in a small concentration of locally frustrating bonds along the $c$ direction, which indeed was 


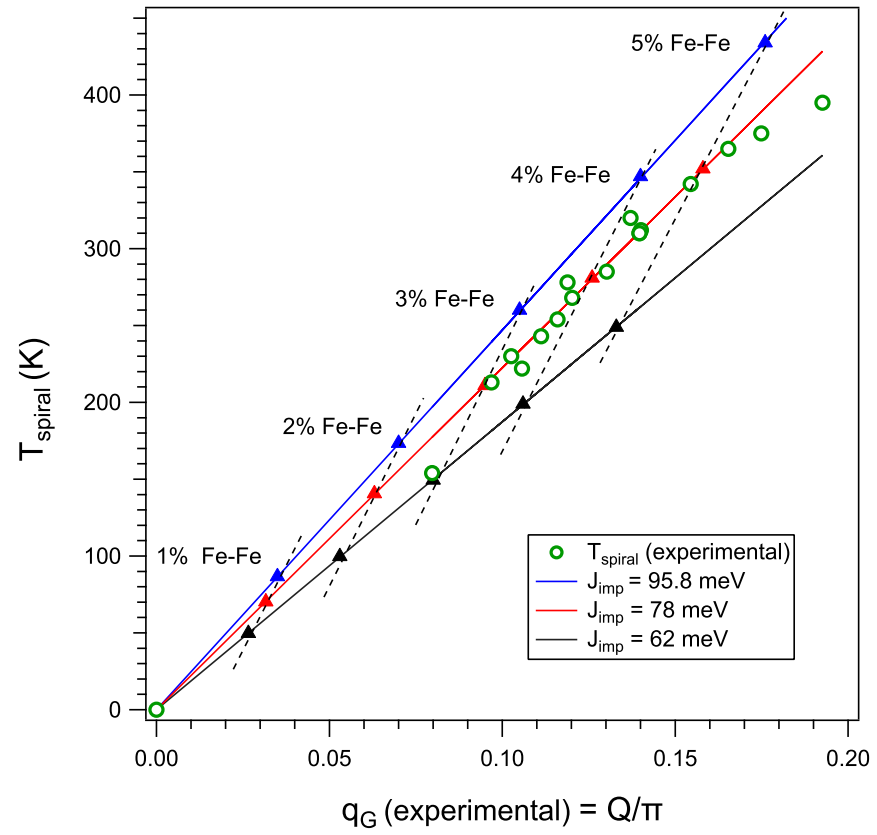

FIG. 1. Experimental data (circles) from Refs. [13,18] for the compound $\mathrm{YBaCuFeO}_{5}$ and similarly layered perovskites with the structure $A B \mathrm{CuFeO}_{5}$. These all contain the magnetic ions $\mathrm{Cu}$ and $\mathrm{Fe}$ in bipyramids that are arranged in layered planes. Different annealing rates lead to varying fractions of bipyramids that host magnetically frustrating $\mathrm{Fe}-\mathrm{Fe}$ pairs instead of low-energy $\mathrm{Fe}-\mathrm{Cu}$ pairs. The transition temperature to the spiral state is plotted versus the spiral wave vector measured at low temperature. Our theory explains the linear relationship between the two and predicts the slope as a function of the three exchange couplings. We plot the predicted slope for different values of the exchange on the driving frustrating bonds, fixing the other two exchanges to the values estimated in Ref. [19]. The larger the frustrating coupling and the higher the fraction of frustrating Fe-Fe bonds in the bipyramids of the perovskite, the larger the transition temperature and the spiral wave vector. Each triplet of triangular symbols joined by a dashed line corresponds to the same concentration of frustrating bonds in our theory.

shown to induce magnetic spiral order in an experimentally relevant window of parameters.

In this paper, we describe and study the general mechanism that renders the ferromagnetic long-range order of classical $X Y$ spins unstable towards spiral order, when a finite fraction of the ferromagnetic interactions is replaced by sufficiently strong antiferromagnetic exchange couplings. In the end, we will confront the theory with experimental data, as shown in Fig. 1, with good quantitative agreement.

The general physical mechanism at work is the following. We consider a geometrically unfrustrated lattice $\Lambda$ in $d>2$ dimensions, hosting isotropic spins with a continuous symmetry. The symmetry is broken spontaneously at low temperatures, which implies the existence of Goldstone modes. Dilute but strong impurity bonds embedded in this lattice can induce local cantings which behave as dipole-type defects with an Ising degree of freedom associated with them. The Goldstone modes mediate an interaction between the defects, decaying as $r^{-d}$ for large separation. Correlations in the distribution of such impurity bonds, e.g., a restriction to bonds that point in a single direction, may ensure a sufficiently nonfrustrated pairwise interaction between these defects so as to favor long-range ferromagnetic order in the orientation of the local cantings. Such long-range Ising order entails a global twist of the ferromagnetic order parameter density, and thus a magnetic spiral, as the local magnetization twists in the same sense across every impurity bond. The wave vector of the resulting magnetic spiral is proportional to the magnetization density of the Ising degrees of freedom and thus to the density of impurity bonds. We will show that such a spiral state is the ground state of the $X Y$ system for a rather wide range of parameters of the impurity bond distribution.

For simplicity, we consider a cubic host lattice $\Lambda$ embedded in three-dimensional Euclidean space with the Cartesian coordinates $x, y$, and $z$. We impose a tetragonal symmetry by choosing the ferromagnetic nearest-neighbor exchange to be $J_{\|}>0$ for couplings in the $x-y$ plane and $J_{\perp}>0$ for bonds oriented along the $z$ axis. We further consider a set of impurity bonds, which form a dilute subset of the nearest-neighbor bonds that are directed along the $c$ direction of the cubic host lattice $\Lambda$. For each impurity bond, the ferromagnetic $J_{\perp}>$ 0 is replaced by the antiferromagnetic exchange coupling $J_{\text {imp }}<0$.

A single impurity bond does not destroy the ferromagnetic long-range order of the ground state. However, it does result in a canting of the classical $X Y$ spins in the vicinity of the impurity bond, provided the local frustration is sufficiently strong, i.e., $\left|J_{\text {imp }}\right| \geqslant J_{\mathrm{c}}$ for some threshold value $J_{\mathrm{c}}>0$. Under these conditions (and with fixed boundary conditions at infinity) the ground state is twofold degenerate, exhibiting either a clockwise or counterclockwise sense of the local canting. At low concentration we can thus associate a corresponding low-energy Ising degree of freedom with every impurity bond. Apart from these discrete soft degrees of freedom, the background ferromagnet hosts low-energy spin-wave excitations. They mediate an effective interaction between the Ising degrees of freedom, which results in an effective classical Ising model with effective two-body interactions of dipolar type. Their algebraic decay at large distance is a direct consequence of the gaplessness of the spin waves. A similar effective interaction results in any system that spontaneously breaks a continuous symmetry, and thus hosts gapless Goldstone modes mediating algebraic interactions between impurity degrees of freedom. The specific case where the impurity bonds form a Bravais superlattice, with a unit cell that is large compared to that of the cubic host lattice $\Lambda$, is analytically tractable, and for certain classes of superlattices we are able to rigorously establish the presence of spiral order.

Although the analysis in this paper assumes ferromagnetic interactions for the host lattice $\Lambda$, we note that our results can be readily extended to any unfrustrated $X Y$ magnet. For example, if the lattice is bipartite and $J_{\text {imp }}$ has sign opposite to $J_{\perp}$, the system can be mapped to the above-described ferromagnet as follows. For every spin, a reference frame is chosen such that the unfrustrated ground state of the impurity-free system corresponds to a ferromagnetic configuration. By virtue of this mapping, the low-energy effective theory presented below extends to this larger class of magnetic insulators.

We emphasize that for the establishment of ferromagnetic order it is central that the impurity bonds not be randomly 
oriented. Otherwise, the pairwise interactions between the associated Ising degrees of freedom would be strongly random in sign, which would most likely lead to spin glass order, as observed in models of dilute, randomly oriented Ising dipoles [20,21]. Since an Ising glass state generally carries no net magnetization, it would not induce a spiral state of the original $X Y$ spins. Also, in the limit of a high density of randomly oriented impurity bonds, one expects long-range spin-glass order (observed directly at the level of the $X Y$ spins), since the model becomes that of a random-bond $X Y$ gauge glass, as was studied by Villain [22-24].

The remainder of this paper is structured as follows. In Sec. II we define the spin lattice model. Section III begins with the case of a single impurity bond. We then consider a small concentration of impurity bonds and derive a mapping to an effective Ising model for low energies. Section IV describes how to find the ground state of the effective Ising model when the impurity bonds realize a superlattice. The effective Ising model is solved by analytical and numerical means. Its solution is then compared to Monte Carlo simulations of a model with the same network of exchange interactions, but in which the classical $X Y$ spins are replaced by classical Heisenberg spins with an additional easy-plane anisotropy. The latter allows for close contact with experimentally realized magnets, such as $\mathrm{YBaCuFeO}_{5}$ and similarly layered perovskites, which are believed to embody the physical ingredients and mechanisms discussed above. In Sec. V we study the analytically tractable case of dilute, randomly placed impurity bonds and conclude that random samples undergo a ferromagnetic ordering of cantings and thus form a spiral phase. Section VI addresses the onset temperature of the spiral phase and predicts it to be proportional to the spiral wave vector. We compare our quantitative predictions with experimental observations. Section VII summarizes our findings and discusses how the general mechanism identified here applies to other systems.

\section{LATTICE HAMILTONIAN FOR CLASSICAL $X Y$ SPINS}

\section{A. Definition of the $X Y$ model}

We consider a magnet of classical spins, described by two-dimensional unit vectors $\hat{\boldsymbol{S}}_{\boldsymbol{r}}$ with $\hat{\boldsymbol{S}}_{\boldsymbol{r}}^{2}=1$. They are located at the sites $\boldsymbol{r}=x \boldsymbol{x}+y \boldsymbol{y}+z \boldsymbol{z}(x, y, z \in \mathbb{Z})$ of a cubic lattice $\Lambda$ made of $|\Lambda|$ sites spanned by the orthonormal unit vectors $\boldsymbol{x}, \boldsymbol{y}$, and $\boldsymbol{z}$ of $\mathbb{R}^{3}$. In most cases we will impose periodic boundary conditions on these classical spins. However, as usual, the choice of boundary conditions does not affect the bulk properties.

We consider a classical Hamiltonian

$$
H_{\mathcal{L}}:=H_{0}+H_{\text {imp }}
$$

containing only nearest-neighbor interactions between spins. Here $\mathcal{L}$ denotes the set of impurity bonds, as we will describe below.

The exchange Hamiltonian in Eq. (2.1a),

$$
H_{0}:=-\frac{1}{2} \sum_{\boldsymbol{r}, \boldsymbol{r}^{\prime} \in \Lambda} J_{\boldsymbol{r}, \boldsymbol{r}^{\prime}}^{(0)} \hat{\boldsymbol{S}}_{\boldsymbol{r}} \cdot \hat{\boldsymbol{S}}_{\boldsymbol{r}^{\prime}}
$$

possesses the translation symmetries of the cubic lattice, since its nearest-neighbor ferromagnetic Heisenberg exchange couplings depend only on the relative position of the spins,

$$
J_{\boldsymbol{r}, \boldsymbol{r}^{\prime}}^{(0)}:=J_{\|} \sum_{\boldsymbol{\alpha}= \pm \boldsymbol{x}, \pm \boldsymbol{y}} \delta_{\boldsymbol{r}, \boldsymbol{r}^{\prime}+\boldsymbol{\alpha}}+J_{\perp} \sum_{\boldsymbol{\alpha}= \pm z} \delta_{\boldsymbol{r}, \boldsymbol{r}^{\prime}+\boldsymbol{\alpha}}=J_{\boldsymbol{r}^{\prime}, \boldsymbol{r}}^{(0)}
$$

The in-plane $\left(J_{\|}\right)$and out-of-plane $\left(J_{\perp}\right)$ couplings are ferromagnetic but can be different, $0<J_{\|} \neq J_{\perp}$, in which case the cubic point-group symmetry is reduced to the tetragonal one.

The contribution from the disorder in Eq. (2.1a),

$$
H_{\mathrm{imp}}:=\left(\left|J_{\mathrm{imp}}\right|+J_{\perp}\right) \sum_{\tilde{\boldsymbol{r}} \in \mathcal{L}} \hat{\boldsymbol{S}}_{\tilde{\boldsymbol{r}}} \cdot \hat{\boldsymbol{S}}_{\tilde{\boldsymbol{r}}+z},
$$

describes the presence of antiferromagnetic impurity bonds. We label the bonds by the coordinate of the end point with the smaller $z$ coordinate. These end points form a subset $\mathcal{L}$ of the points of the cubic host lattice $\Lambda$. This term breaks the lattice translational symmetry. On all impurity bonds the ferromagnetic $J_{\perp}>0$ is replaced by the antiferromagnetic coupling $J_{\text {imp }}<0$, inducing local frustration. The Hamiltonian (2.1a) is invariant under any rotation of all spins by the same orthogonal $2 \times 2$ matrix, i.e., $H_{\mathcal{L}}$ has a global $\mathrm{O}(2)$ symmetry.

\section{B. Impurity-free case}

Here we consider an impurity-free system, i.e., an empty set $\mathcal{L}$,

$$
H_{\mathcal{L}}=H_{0}
$$

The ground state is ferromagnetic with all spins parallel. We choose the polar parametrization

$$
\hat{\boldsymbol{S}}_{\boldsymbol{r}}:=\cos \theta_{\boldsymbol{r}} \hat{\boldsymbol{x}}+\sin \theta_{\boldsymbol{r}} \hat{\boldsymbol{y}}
$$

with the orthonormal basis $\hat{\boldsymbol{x}}$ and $\hat{\boldsymbol{y}}$ of $\mathbb{R}^{2}$. In this polar representation,

$$
H_{0}=-\frac{1}{2} \sum_{\boldsymbol{r}, \boldsymbol{r}^{\prime} \in \Lambda} J_{\boldsymbol{r}, \boldsymbol{r}^{\prime}}^{(0)} \cos \left(\theta_{\boldsymbol{r}}-\theta_{\boldsymbol{r}^{\prime}}\right)
$$

has a ferromagnetic ground state defined by

$$
\theta_{r}^{\text {ferro }} \equiv \text { const }
$$

for all lattice sites $\boldsymbol{r}$. The invariance of $H_{\mathcal{L}}$ under any global $\mathrm{O}(2)$ symmetry then becomes the invariance under the symmetry transformation

$$
\theta_{\boldsymbol{r}} \mapsto \epsilon \theta_{\boldsymbol{r}}+\Theta
$$

where $\epsilon= \pm 1$ and $\Theta \in[0,2 \pi$ [ are arbitrary numbers independent of $\boldsymbol{r}$. The angle $\Theta \in[0,2 \pi$ [ parametrizes a proper rotation in the connected Lie group $\mathrm{SO}(2)$. The choice $\epsilon=-1$ corresponds to an improper rotation, i.e., an orthogonal matrix in $\mathrm{O}(2)$ with negative determinant

At low temperatures $T \ll J_{\perp}, J_{\|}$, we can use the spinwave approximation, which assumes that the deviations from the ferromagnetic ground state (2.5) are small. In that case, the Hamiltonian (2.4) can be expanded to quadratic order in 
the angle differences,

$$
\begin{aligned}
H_{0} & \approx E_{\mathrm{FM}}+\frac{1}{4} \sum_{\boldsymbol{r}, \boldsymbol{r}^{\prime} \in \Lambda} J_{\boldsymbol{r}, \boldsymbol{r}^{\prime}}^{(0)}\left(\theta_{\boldsymbol{r}}-\theta_{\boldsymbol{r}^{\prime}}\right)^{2} \\
& =E_{\mathrm{FM}}+\frac{1}{2} \sum_{\boldsymbol{r}, \boldsymbol{r}^{\prime} \in \Lambda} \theta_{\boldsymbol{r}} D_{\boldsymbol{r}, \boldsymbol{r}^{\prime}}^{(0)} \theta_{\boldsymbol{r}^{\prime}},
\end{aligned}
$$

where

$$
E_{\mathrm{FM}} \equiv-\frac{1}{2} \sum_{\boldsymbol{r}, \boldsymbol{r}^{\prime} \in \Lambda} J_{\boldsymbol{r}, \boldsymbol{r}^{\prime}}^{(0)}
$$

is the energy of the ferromagnetic ground state and

$$
\begin{aligned}
D_{\boldsymbol{r}, \boldsymbol{r}^{\prime}}^{(0)} & :=\left(\sum_{\boldsymbol{r}^{\prime} \in \Lambda} J_{\boldsymbol{r}, \boldsymbol{r}^{\prime \prime}}^{(0)}\right) \delta_{\boldsymbol{r}, \boldsymbol{r}^{\prime}}-J_{\boldsymbol{r}, \boldsymbol{r}^{\prime}}^{(0)} \\
& =\left(4 J_{\|}+2 J_{\perp}\right) \delta_{\boldsymbol{r}, \boldsymbol{r}^{\prime}}-J_{\boldsymbol{r}, \boldsymbol{r}^{\prime}}^{(0)} \\
& =D_{\boldsymbol{r}^{\prime}, \boldsymbol{r}}^{(0)} \equiv D_{\boldsymbol{r}^{\prime}-\boldsymbol{r}}^{(0)} \equiv D_{\boldsymbol{r}-\boldsymbol{r}^{\prime}}^{(0)}
\end{aligned}
$$

is the symmetric spin-wave kernel. It only depends on the difference $\boldsymbol{r}^{\prime}-\boldsymbol{r}$, which we henceforth use as the only subscript.

The spin wave encoded by the angle $\theta_{\boldsymbol{r}}$ is the gapless Goldstone mode associated with the spontaneous breaking of the $\mathrm{O}(2)$ spin symmetry by the ferromagnetic ground state. On general grounds, its Fourier mode $\theta_{\boldsymbol{k}}$ is expected to disperse quadratically with momentum as $\boldsymbol{k}$ approaches the center of the magnetic Brillouin zone, $\boldsymbol{k}=0$. Accordingly, the response to a local perturbation at $\boldsymbol{r}$ and temperature $T$ is governed by the Green's function

$$
T^{-1}\left\langle\theta_{\boldsymbol{r}} \theta_{\boldsymbol{r}^{\prime}}\right\rangle \equiv\left(D^{(0)}\right)_{\boldsymbol{r}, \boldsymbol{r}^{\prime}}^{-1},
$$

which decays like $\left|\boldsymbol{r}-\boldsymbol{r}^{\prime}\right|^{-1}$ at large separations $\left|\boldsymbol{r}-\boldsymbol{r}^{\prime}\right| \gg \mathfrak{a}$, where $\mathfrak{a}$ is of the order of the lattice spacing. We close Sec. II by establishing a few important properties obeyed by the spinwave kernel $D^{(0)}$.

We observe that $D_{r}^{(0)}$ obeys

$$
\sum_{r^{\prime} \in \Lambda} D_{r^{\prime}}^{(0)}=0
$$

This is a consequence of spin rotational symmetry, which implies that any global orthogonal transformation (2.6) leaves the bilinear form (2.7a) invariant. Moreover, if we impose that the angles $\theta_{r}$ obey periodic boundary conditions, we then have the Fourier transform

$$
\begin{aligned}
D_{\boldsymbol{k}}^{(0)} & :=\frac{1}{|\Lambda|} \sum_{\boldsymbol{r} \in \Lambda} e^{-i \boldsymbol{k} \cdot \boldsymbol{r}} D_{\boldsymbol{r}}^{(0)} \\
& =2 J_{\|}\left(2-\cos k_{x}-\cos k_{y}\right)+2 J_{\perp}\left(1-\cos k_{z}\right)
\end{aligned}
$$

for any $\boldsymbol{k}$ belonging to the Brillouin zone of the host cubic lattice $\Lambda$. We will denote this Brillouin zone by $\operatorname{BZ}(\Lambda)$. Finally, it is convenient to introduce the inverse of the spinwave kernel $D^{(0)}$ as the Green's function $G^{(0)}$, which satisfies

$$
\sum_{\boldsymbol{r}^{\prime} \in \Lambda} G_{\boldsymbol{r}-\boldsymbol{r}^{\prime}}^{(0)} D_{\boldsymbol{r}^{\prime}-\boldsymbol{r}^{\prime \prime}}^{(0)}=\delta_{\boldsymbol{r}, \boldsymbol{r}^{\prime \prime}} \forall \boldsymbol{r}, \boldsymbol{r}^{\prime \prime} .
$$

Due to the zero mode (2.9), $G_{\boldsymbol{r}-\boldsymbol{r}^{\prime}}^{(0)}$ is defined up to a constant, which we fix by requiring that

$$
\sum_{r \in \Lambda} G_{r}^{(0)}=0
$$

such that both $G^{(0)}$ and $D^{(0)}$ annihilate constant functions. As the inverse of a symmetric kernel, $G_{r-r^{\prime}}^{(0)}$ is symmetric,

$$
G_{\boldsymbol{r}-\boldsymbol{r}^{\prime}}^{(0)}=G_{\boldsymbol{r}^{\prime}-\boldsymbol{r}}^{(0)} .
$$

Imposing periodic boundary conditions, we have

$$
G_{r}^{(0)}=\frac{1}{|\Lambda|} \sum_{k \in \mathrm{BZ}(\Lambda) \backslash\{0\}} \frac{e^{i \boldsymbol{k} \cdot \boldsymbol{r}}}{D_{\boldsymbol{k}}^{(0)}} .
$$

The asymptotic large distance behavior of the Green's function is

$$
\begin{aligned}
G_{r}^{(0)} \underset{|\boldsymbol{r}| \rightarrow \infty}{\approx} \int \frac{d^{3} \boldsymbol{k}}{(2 \pi)^{3}} \frac{e^{i \boldsymbol{k} \cdot \boldsymbol{r}}}{J_{\|}\left(k_{x}^{2}+k_{y}^{2}\right)+J_{\perp} k_{z}^{2}} \\
\quad=\frac{1}{4 \pi \sqrt{J_{\|}}} \frac{1}{\sqrt{J_{\perp}\left(x^{2}+y^{2}\right)+J_{\|} z^{2}}} .
\end{aligned}
$$

On the right-hand side of Eq. (2.12), we recognize the threedimensional Coulomb potential for the rescaled coordinates

$$
\bar{x}=\sqrt{J_{\perp}} x, \quad \bar{y}=\sqrt{J_{\perp}} y, \quad \bar{z}=\sqrt{J_{\|}} z .
$$

We will see in the next section that impurities couple to each other through the combination of Green's functions

$$
\begin{aligned}
\Gamma_{\boldsymbol{r}}^{(0)} & :=2 G_{\boldsymbol{r}}^{(0)}-G_{\boldsymbol{r}+z}^{(0)}-G_{\boldsymbol{r}-\boldsymbol{z}}^{(0)} \\
& =\frac{1}{|\Lambda|} \sum_{\boldsymbol{k} \in \mathrm{BZ}(\Lambda) \backslash\{0\}} \hat{\Gamma}_{\boldsymbol{k}}^{(0)} e^{i \boldsymbol{k} \cdot \boldsymbol{r}}
\end{aligned}
$$

with the Fourier transform of $\Gamma_{\boldsymbol{r}}^{(0)}$,

$$
\hat{\Gamma}_{k \neq \mathbf{0}}^{(0)}:=\frac{1-\cos k_{z}}{J_{\|}\left(2-\cos k_{x}-\cos k_{y}\right)+J_{\perp}\left(1-\cos k_{z}\right)} .
$$

Note that $\hat{\Gamma}_{\boldsymbol{k}=\mathbf{0}}^{(0)}$ does not enter the sum. It is therefore convenient to define

$$
\hat{\Gamma}_{k=\mathbf{0}}^{(0)}:=0 .
$$

Asymptotically, $\Gamma_{r}^{(0)}$ decays algebraically, like a dipolar interaction with opposite sign,

$$
\Gamma_{\boldsymbol{r}}^{(0)} \underset{|r| \rightarrow \infty}{\approx}-\partial_{z}^{2} G_{\boldsymbol{r}}^{(0)} \approx \frac{\sqrt{J_{\|}}}{4 \pi} \frac{|\overline{\boldsymbol{r}}|^{2}-3 \bar{z}^{2}}{|\overline{\boldsymbol{r}}|^{5}},
$$

where we use the notation of Eq. (2.13).

\section{MAPPING TO AN EFFECTIVE ISING HAMILTONIAN}

\section{A. Periodic boundary conditions}

We are ultimately interested in describing states with a spiraling magnetic order, where the angles of the local magnetization grow linearly with distance. However, before dealing with this possibility, we first analyze a situation anticipating no spiraling. In this case we can impose periodic boundary conditions on the spin angles. 
We recall that the set of all directed impurity bonds defines the set $\mathcal{L} \subset \Lambda$ consisting of all the sites $\tilde{\boldsymbol{r}} \in \Lambda$ such that $\langle\tilde{\boldsymbol{r}}, \tilde{\boldsymbol{r}}+\boldsymbol{z}\rangle$ is a directed impurity bond. We anticipate that the twist angles across bonds are relatively small, except on the impurity bonds. We therefore make the approximation

$$
\begin{aligned}
H_{\mathcal{L}} \approx & E_{\mathrm{FM}}+\frac{1}{2} \sum_{\boldsymbol{r}, \boldsymbol{r}^{\prime} \in \Lambda} \theta_{\boldsymbol{r}} D_{\boldsymbol{r}-\boldsymbol{r}^{\prime}}^{(0)} \theta_{\boldsymbol{r}^{\prime}} \\
& -\sum_{\tilde{\boldsymbol{r}} \in \mathcal{L}}\left[\frac{J_{\perp}}{2}\left(\Delta \theta_{\tilde{\boldsymbol{r}}}\right)^{2}-\left|J_{\mathrm{imp}}\right| \cos \Delta \theta_{\tilde{\boldsymbol{r}}}\right],
\end{aligned}
$$

where $\Delta \theta_{\tilde{\boldsymbol{r}}}$ is the canting angle across the impurity bond,

$$
\Delta \theta_{\tilde{\boldsymbol{r}}} \equiv \theta_{\tilde{\boldsymbol{r}}}-\theta_{\tilde{\boldsymbol{r}}+z} .
$$

This approximation is certainly valid on the majority of bonds in the limit of a dilute concentration of impurity bonds,

$$
n_{\text {imp }}:=\frac{|\mathcal{L}|}{|\Lambda|} \ll 1,
$$

which we will assume from now on.

We are going to establish under what conditions there is a configuration of angles other than the ferromagnetic one that minimizes the energy (3.1a) in the presence of impurity bonds anchored on the set $\mathcal{L}$. First, we keep fixed all the angles $\theta_{\tilde{r}}$ and $\theta_{\tilde{\boldsymbol{r}}+z}$ with $\tilde{\boldsymbol{r}} \in \mathcal{L}$ and integrate out the angles on all other sites, i.e., on all sites from $\Lambda \backslash(\mathcal{L} \cup \mathcal{L}+z)$. Within our spinwave approximation, and treating the angles as noncompact variables, this can be done exactly (at any temperature where the spin-wave approximation is justified) since those angular variables enter the Hamiltonian quadratically. Here we carry out the calculation at $T=0$ by solving the saddle-point equations for all angles on the sites $\Lambda \backslash(\mathcal{L} \cup \mathcal{L}+z)$. In this way, we will find the angles $\theta_{\boldsymbol{r}}$, as well as an effective Hamiltonian, expressed solely in terms of the angles $\left\{\theta_{\tilde{r}}, \tilde{\boldsymbol{r}} \in \mathcal{L} \cup \mathcal{L}+\boldsymbol{z}\right\}$.

The minimization over all $\boldsymbol{r} \in \Lambda \backslash(\mathcal{L} \cup \mathcal{L}+\boldsymbol{z})$ requires

$$
\sum_{\boldsymbol{r}^{\prime} \in \Lambda} D_{\boldsymbol{r}-\boldsymbol{r}^{\prime}}^{(0)} \theta_{\boldsymbol{r}^{\prime}}=0 .
$$

We supplement the set (3.3a) of linear equations by

$$
\sum_{\boldsymbol{r}^{\prime} \in \Lambda} D_{\tilde{\boldsymbol{r}}-\boldsymbol{r}^{\prime}}^{(0)} \theta_{\boldsymbol{r}^{\prime}}=\chi_{\tilde{r}}
$$

whereby $\tilde{\boldsymbol{r}} \in \mathcal{L} \cup \mathcal{L}+z$ runs over all sites in $\Lambda$ that are end points of an impurity bond (two sites per impurity bond). Here $\chi_{\tilde{r}}$ has to be chosen in such a way that the saddle-point equations (3.3) are satisfied as $\theta_{\tilde{\boldsymbol{r}}}$ and $\theta_{\tilde{\boldsymbol{r}}+z}$ take their prescribed values.

Inverting Eqs. (3.3a) and (3.3b) for any $\boldsymbol{r} \in \Lambda$, we obtain

$$
\theta_{\boldsymbol{r}}=\theta_{0}+\sum_{\tilde{\boldsymbol{r}}^{\prime} \in \mathcal{L} \cup \mathcal{L}+z} G_{\boldsymbol{r}, \tilde{r}^{\prime}}^{(0)} \chi_{\tilde{r}^{\prime}}
$$

where $\theta_{0} \in[0,2 \pi$ [ is the angle of the magnetization far away from impurities. Restricting ourselves to the subset $\tilde{\boldsymbol{r}} \in \mathcal{L} \cup$ $\mathcal{L}+\boldsymbol{z}$, Eq. (3.4) can be inverted to yield

$$
\chi_{\tilde{r}}=\sum_{\tilde{\boldsymbol{r}}^{\prime} \in \mathcal{L} \cup \mathcal{L}+z} \tilde{G}_{\tilde{\boldsymbol{r}}, \tilde{r}^{\prime}}^{(0)-1}\left(\theta_{\tilde{\boldsymbol{r}}^{\prime}}-\theta_{0}\right),
$$

where $\tilde{G}^{(0)}$ is the $2|\mathcal{L}| \times 2|\mathcal{L}|$ matrix obtained by restricting $G_{r, r^{\prime}}^{(0)}$ to the sites belonging to the impurity bonds $\left[\tilde{G}_{\tilde{r}, \tilde{r}^{\prime}}^{(0)} \equiv\right.$
$\left.G_{\tilde{r}, \tilde{r}^{\prime}}^{(0)}\right]$. Combining Eqs. (3.4) and (3.5), we obtain, for any $r \in \Lambda$,

$$
\theta_{\boldsymbol{r}}=\theta_{0}+\sum_{\tilde{\boldsymbol{r}}^{\prime}, \tilde{\boldsymbol{r}}^{\prime \prime} \in \mathcal{L} \cup \mathcal{L}+z} G_{\boldsymbol{r}, \tilde{\boldsymbol{r}}^{\prime}}^{(0)} \tilde{G}_{\tilde{\boldsymbol{r}}^{\prime}, \tilde{r}^{\prime \prime}}^{(0)-1}\left(\theta_{\tilde{\boldsymbol{r}}^{\prime \prime}}-\theta_{0}\right)
$$

The algebraic decay of $G_{r}^{(0)}$ ensures that the distortions in the angular pattern also have algebraic tails.

Second, we evaluate the energy (3.1a) at the saddle point (3.6). We thereby obtain the effective energy

$$
\begin{aligned}
H_{\mathrm{eff}}\left(\left\{\theta_{\tilde{\boldsymbol{r}}}, \theta_{\tilde{\boldsymbol{r}}+z}, \tilde{\boldsymbol{r}} \in \mathcal{L}\right\}\right) & =\frac{1}{2} \sum_{\tilde{\boldsymbol{r}}, \tilde{\boldsymbol{r}}^{\prime} \in \mathcal{L} \cup \mathcal{L}+z} \theta_{\tilde{r}} \tilde{G}_{\tilde{\boldsymbol{r}}, \tilde{\boldsymbol{r}}^{\prime}}^{(0)-1} \theta_{\tilde{\boldsymbol{r}}^{\prime}} \\
& -\sum_{\tilde{\boldsymbol{r}} \in \mathcal{L}}\left[\frac{J_{\perp}}{2}\left(\theta_{\tilde{\boldsymbol{r}}+z}-\theta_{\tilde{\boldsymbol{r}}}\right)^{2}-\left|J_{\mathrm{imp}}\right| \cos \left(\theta_{\tilde{\boldsymbol{r}}+z}-\theta_{\tilde{\boldsymbol{r}}}\right)\right],
\end{aligned}
$$

where we have assumed that the magnetization far away from the impurities are oriented along $\theta_{0}=0$, making use of the fact that the constant $\theta_{0}$ can be chosen freely, since rotating all spins by the same angle does not affect the energy. In what follows, we will drop such additive constants. Note that the derivation of the effective action (3.7), starting from the Hamiltonian (3.1a), is exact at any temperature, if we use the Gaussian approximation and treat the domain of the angles $\theta_{r}$ as noncompact, ignoring that the energy is in fact $2 \pi$ periodic in the angles.

For the third and last step we assume $T=0$. We minimize the effective action (3.7) with respect to the impurity bond angles $\theta_{\tilde{\boldsymbol{r}}}$ and $\theta_{\tilde{\boldsymbol{r}}+z}$ with $\tilde{\boldsymbol{r}} \in \mathcal{L}$. This can be done exactly for a single impurity bond and approximately in the case of a dilute set of impurities.

\section{Case of a single impurity bond}

In the case of a single impurity bond $\langle\tilde{\boldsymbol{r}}, \tilde{\boldsymbol{r}}+\boldsymbol{z}\rangle$ represented by the single site $\mathcal{L}=\{\tilde{\boldsymbol{r}}\}$, the inverse of the $2 \times 2$ symmetric matrix with elements $\tilde{G}_{\tilde{r}, \tilde{r}}^{(0)}, \tilde{G}_{\tilde{\boldsymbol{r}}, \tilde{r}+z}^{(0)}, \tilde{G}_{\tilde{\boldsymbol{r}}+z, \tilde{\boldsymbol{r}}}^{(0)}$, and $\tilde{G}_{\tilde{\boldsymbol{r}}+z, \tilde{\boldsymbol{r}}+z}^{(0)}$ is

$$
\tilde{G}_{\boldsymbol{r}, \boldsymbol{r}^{\prime}}^{(0)-1}=\frac{G_{0}^{(0)} \delta_{\boldsymbol{r}, \boldsymbol{r}^{\prime}}-G_{z}^{(0)}\left(\delta_{\boldsymbol{r}, \boldsymbol{r}^{\prime}+z}+\delta_{\boldsymbol{r}, \boldsymbol{r}^{\prime}-z}\right)}{\left(G_{\mathbf{0}}^{(0)}\right)^{2}-\left(G_{z}^{(0)}\right)^{2}},
$$

where $\boldsymbol{r}, \boldsymbol{r}^{\prime} \in\{\tilde{\boldsymbol{r}}, \tilde{\boldsymbol{r}}+\boldsymbol{z}\}$. If we use Eq. (3.8) in the Hamiltonian (3.7), we find the effective energy

$$
\begin{aligned}
H_{\mathrm{eff}}^{(1)}\left(\theta_{\tilde{\boldsymbol{r}}}, \theta_{\tilde{\boldsymbol{r}}+z}\right)= & \frac{1}{4} \frac{\left(\theta_{\tilde{\boldsymbol{r}}}+\theta_{\tilde{\boldsymbol{r}}+z}\right)^{2}}{G_{\mathbf{0}}^{(0)}+G_{z}^{(0)}}+\frac{1}{4} \frac{\left(\theta_{\tilde{\boldsymbol{r}}}-\theta_{\tilde{\boldsymbol{r}}+z}\right)^{2}}{G_{\mathbf{0}}^{(0)}-G_{z}^{(0)}} \\
& -\frac{J_{\perp}}{2}\left(\theta_{\tilde{\boldsymbol{r}}}-\theta_{\tilde{\boldsymbol{r}}+z}\right)^{2}+\left|J_{\mathrm{imp}}\right| \cos \left(\theta_{\tilde{\boldsymbol{r}}}-\theta_{\tilde{\boldsymbol{r}}+z}\right) .
\end{aligned}
$$

The center-of-mass angle $\theta_{\tilde{\boldsymbol{r}}}+\theta_{\tilde{\boldsymbol{r}}+z}$ and the relative angle $\theta_{\tilde{\boldsymbol{r}}}-$ $\theta_{\tilde{\boldsymbol{r}}+z}$ are decoupled in $H_{\mathrm{eff}}^{(1)}\left(\theta_{\tilde{\boldsymbol{r}}}, \theta_{\tilde{\boldsymbol{r}}+z}\right)$. The effective Hamiltonian (3.9) is invariant under the transformation

$$
\theta_{\tilde{\boldsymbol{r}}}+\theta_{\tilde{\boldsymbol{r}}+z} \mapsto \theta_{\tilde{\boldsymbol{r}}}+\theta_{\tilde{\boldsymbol{r}}+z}, \quad \theta_{\tilde{\boldsymbol{r}}}-\theta_{\tilde{\boldsymbol{r}}+z} \mapsto-\left(\theta_{\tilde{\boldsymbol{r}}}-\theta_{\tilde{\boldsymbol{r}}+z}\right) .
$$

This symmetry is inherited from the fact that the Hamiltonian (3.7) is invariant under the inversion symmetry with respect to the bond center $\boldsymbol{R}=\tilde{\boldsymbol{r}}+(\boldsymbol{z} / 2)$.

Minimization over $\theta_{\tilde{r}}+\theta_{\tilde{r}+z}$ imposes the condition that the two angular distortions away from the asymptotic $\theta_{0}$ on either 
(a)

(b)

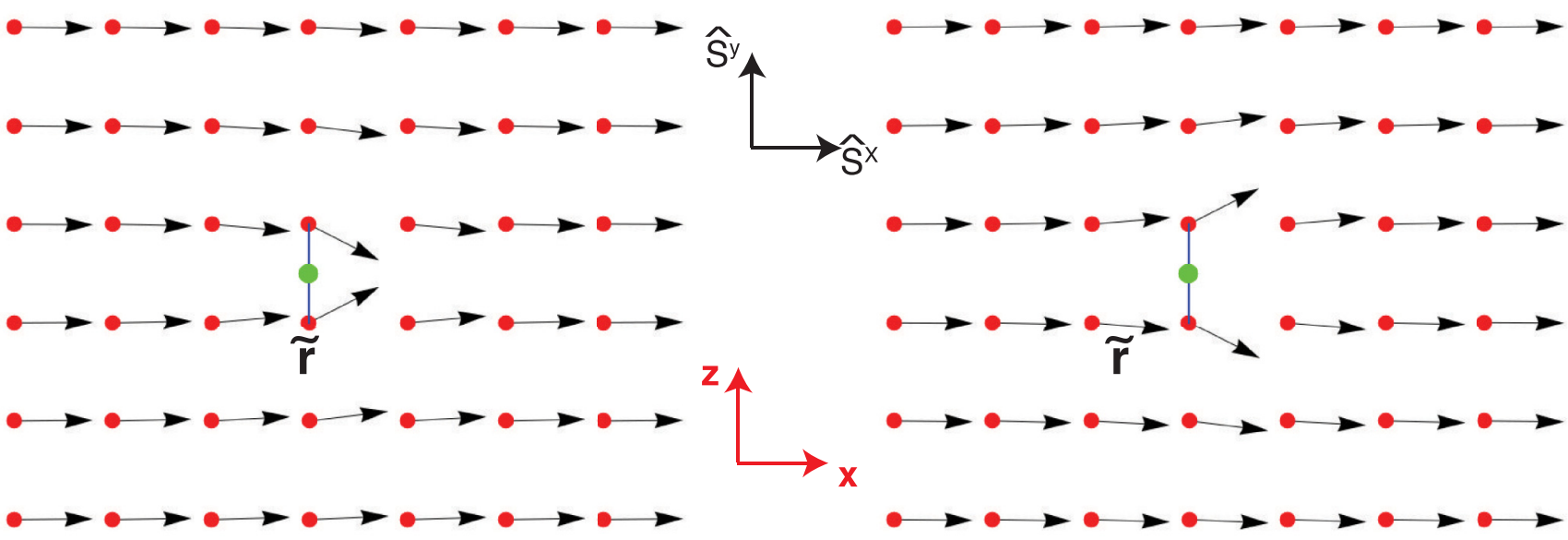

FIG. 2. Cantings of the spins induced by a single strong impurity bond with $\left|J_{\text {imp }}\right|>J_{\mathrm{c}}$. The two degenerate ground states correspond to the two solutions (a) $\sigma_{\tilde{r}}=+1$ and (b) $\sigma_{\tilde{r}}=-1$ of Eq. (3.15). Red circles indicate sites in the $x$ - $z$ plane (red reference frame). The black arrows represent the $\hat{S}^{x}$ and $\hat{S}^{y}$ components of the spins (black reference frame). The blue line indicates a frustrating antiferromagnetic bond embedded in the network of ferromagnetic couplings. The green circles indicate the inversion center with respect to which the Hamiltonian is symmetric. The operation of inversion maps the spin configurations (a) and (b) onto each other.

side of the impurity bond are opposite,

$$
\theta_{\tilde{r}}=-\theta_{\tilde{r}+z} .
$$

The remaining degree of freedom is the canting angle across the impurity bond,

$$
\Delta \theta_{\tilde{\boldsymbol{r}}} \equiv \theta_{\tilde{\boldsymbol{r}}}-\theta_{\tilde{\boldsymbol{r}}+z},
$$

with $-\pi<\Delta \theta_{\tilde{r}} \leqslant \pi$, in terms of which the effective restricted Hamiltonian becomes

$$
H_{\text {eff } / \text { res }}^{(1)}\left(\Delta \theta_{\tilde{\boldsymbol{r}}}\right):=\frac{1}{2} J_{\mathrm{c}}\left(\Delta \theta_{\tilde{\boldsymbol{r}}}\right)^{2}+\left|J_{\mathrm{imp}}\right| \cos \left(\Delta \theta_{\tilde{\boldsymbol{r}}}\right),
$$

where we have introduced the shorthand notation

$$
J_{\mathrm{c}}:=\frac{1}{2\left(G_{0}^{(0)}-G_{z}^{(0)}\right)}-J_{\perp} .
$$

The coupling $J_{\mathrm{c}}$ depends parametrically on $J_{\|}$and $J_{\perp}$. The rationale for the subscript in $J_{\mathrm{c}}$ is the following. For small $\left|J_{\text {imp }}\right|$, i.e., $\left|J_{\text {imp }}\right|<J_{\mathrm{c}}, H_{\text {eff } / \text { res }}^{(1)}\left(\Delta \theta_{\tilde{r}}\right)$ has a single minimum at

$$
\Delta \theta_{\tilde{r}}=0 .
$$

However, for $\left|J_{\text {imp }}\right|>J_{\mathrm{c}}, H_{\text {eff } / \text { res }}^{(1)}\left(\Delta \theta_{\tilde{r}}\right)$ develops a double well with two degenerate minima. The two degenerate minima occur at the relative canting angles

$$
\Delta \theta_{\tilde{r}}=\sigma_{\tilde{r}} \Delta \theta,
$$

with $\sigma_{\tilde{r}}= \pm 1$ and $\Delta \theta$ being the positive solution of

$$
\Delta \theta=\frac{\left|J_{\text {imp }}\right|}{J_{\mathrm{c}}} \sin \Delta \theta .
$$

When $\left|J_{\mathrm{imp}}\right|>J_{\mathrm{c}}$ and the temperature $T$ is sufficiently small, namely,

$$
k_{\mathrm{B}} T \ll J_{\mathrm{c}}-\left|J_{\mathrm{imp}}\right| \cos (\Delta \theta),
$$

thermal fluctuations around the minima of the double well are small. We will thus call $\left|\Delta \theta_{\tilde{r}}\right|$ a hard degree of freedom, while we refer to the Ising variable $\operatorname{sgn}\left(\Delta \theta_{\tilde{r}}\right)=\sigma_{\tilde{r}}$ as a soft degree of freedom. This terminology is motivated by expanding about the minimum of the double potential well $\sigma_{\tilde{r}} \Delta \theta$, which is closest to $\Delta \theta_{\tilde{r}}$,

$$
\begin{aligned}
& H_{\text {eff } / \text { res }}^{(1)}\left(\Delta \theta_{\tilde{\boldsymbol{r}}}\right)-H_{\mathrm{eff} / \mathrm{res}}^{(1)}(\Delta \theta) \\
& \quad=\frac{1}{2}\left[J_{\mathrm{c}}-\left|J_{\text {imp }}\right| \cos (\Delta \theta)\right]\left(\left|\Delta \theta_{\tilde{\boldsymbol{r}}}\right|-\Delta \theta\right)^{2}+\cdots .
\end{aligned}
$$

When the temperature is small compared to the curvature at the two minima, thermal fluctuations of the hard degree of freedom $\left|\Delta \theta_{\tilde{r}}\right|$ are much smaller than fluctuations due to the soft degree of freedom $\sigma_{\tilde{r}}$. We will thus ignore the latter in the range (3.16) of temperatures. If we use the values of the relative angle (3.1b) at the pair of minima (3.15) in combination with Eqs. (3.11) and (3.6) with $\theta_{0}=0$, we obtain the two canting patterns shown in Fig. 2.

The combination $2\left(G_{\mathbf{0}}^{(0)}-G_{z}^{(0)}\right)=\Gamma_{r=0}^{(0)}$ entering $J_{\mathrm{c}}$ in Eq. (3.13b) can be expressed with the help of Eq. (2.14a) as

$$
\begin{aligned}
\Gamma_{r=\mathbf{0}}^{(0)} & =2\left(G_{\mathbf{0}}^{(0)}-G_{z}^{(0)}\right) \\
& =\frac{1}{J_{\mathrm{c}}+J_{\perp}} \\
& =\frac{1}{|\Lambda|} \sum_{\boldsymbol{k} \in \mathrm{BZ}(\Lambda) \backslash\{\mathbf{0}\}} \frac{\left(1-\cos k_{z}\right)}{J_{\|}\left(2-\cos k_{x}-\cos k_{y}\right)+J_{\perp}\left(1-\cos k_{z}\right)} .
\end{aligned}
$$

For isotropic couplings $J_{\|}=J_{\perp}=J$, this evaluates to

$$
\Gamma_{\mathbf{0}}^{(0)}=\frac{1}{3 J},
$$

which yields the critical coupling

$$
J_{\mathrm{c}}=2 J \text {. }
$$

For general couplings with the ratio

$$
\alpha \equiv \frac{J_{\|}}{J_{\perp}},
$$




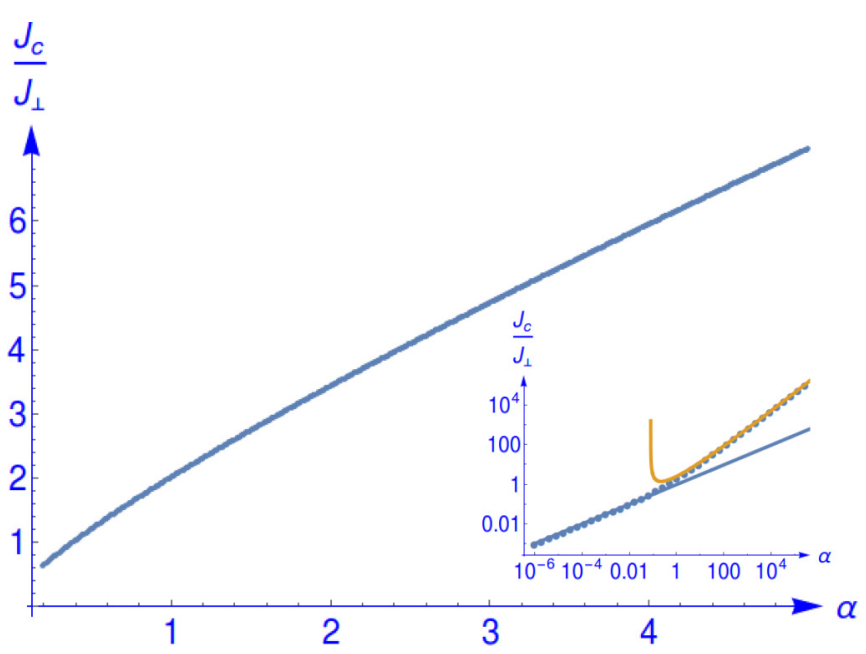

FIG. 3. Critical impurity bond strength $J_{\mathrm{c}}$ [Eq. (3.13b)] (blue dots), as a function of the ratio of couplings $\alpha \equiv J_{\|} / J_{\perp}$. The inset shows the same function on a logarithmic plot. For strong anisotropies, one finds the asymptotics $J_{\mathrm{c}}(\alpha \ll 1) / J_{\perp} \approx$ $C_{1} \sqrt{\alpha}$ (blue line) and $J_{\mathrm{c}}(\alpha \gg 1) / J_{\perp} \approx 2 \pi \alpha /\left[\ln (\alpha)+C_{2}\right]$ (yellow curve), where $C_{1}=\int_{0}^{\pi} d x d y \sqrt{\sin ^{2} x+\sin ^{2} y} / \pi^{2} \approx 0.958$ and $C_{2}=$ $(5 \ln 2-1) \approx 2.47$.

we plot the threshold value of $J_{\mathrm{c}} / J_{\perp}$ in Fig. 3 together with asymptotic expressions that become valid in the limit of strong anisotropy.

The spontaneous canting induced by a single impurity bond is a special case of a so-called canted local state, as described by Villain in Ref. [25]. He pointed out that in a magnet with continuous spin symmetry and a pristine collinear long-range-ordered ground state, local imperfections such as clusters of frustrating exchange couplings can cause spins in their neighborhood to cant. Thereby, the canting angle only decays algebraically with distance from the impurity. As already noted by Villain for random bond impurities in a collinear magnet, a critical strength of the impurity bond is necessary to induce canting. Since canting spontaneously breaks the inversion symmetry of the pristine ground state in our problem, there is a degenerate set of canted local states associated with each impurity. This is in contrast to frustrated $\mathrm{O}(N)$-symmetric magnets with noncollinear ground states and no inversion symmetry for which any local deviation in the bond strength induces a unique canting deformation, with similar algebraic decay of canting angles. Those algebraic tails destroy long-range order even if the disorder is very weak (see Ref. [26]).

\section{B. Case of a dilute set of impurity bonds}

When the density of impurity bonds is finite, we cannot rely on the explicit representation (3.8). However, at a small impurity concentration, the interaction between the angles on the same impurity bond is much stronger than the coupling between angles on different impurity bonds. It thus makes sense to split $\tilde{G}^{(0)}$ into a bond-local term [with the inverse on every bond given by Eq. (3.8)] and a bond-off-diagonal term according to

$$
\tilde{G}^{(0)} \equiv \tilde{G}_{\mathrm{loc}}^{(0)}+\tilde{G}_{\text {nonloc }}^{(0)}
$$

and to approximate its inverse as

$$
\tilde{G}^{(0)-1} \approx \tilde{G}_{\mathrm{loc}}^{(0)-1}-\tilde{G}_{\mathrm{loc}}^{(0)-1} \tilde{G}_{\mathrm{nonloc}}^{(0)} \tilde{G}_{\mathrm{loc}}^{(0)-1} .
$$

This yields the Hamiltonian

$$
H_{\mathrm{eff}}\left(\left\{\theta_{\tilde{\boldsymbol{r}}}, \theta_{\tilde{\boldsymbol{r}}+z}, \tilde{\boldsymbol{r}} \in \mathcal{L}\right\}\right) \approx \sum_{\tilde{\boldsymbol{r}} \in \mathcal{L}} H_{\mathrm{eff}}^{(1)}\left(\theta_{\tilde{\boldsymbol{r}}}, \theta_{\tilde{\boldsymbol{r}}+z}\right)-\frac{1}{2} \sum_{\tilde{\boldsymbol{r}}, \tilde{\boldsymbol{r}}^{\prime} \in \mathcal{L} \cup \mathcal{L}+z} \theta_{\tilde{\boldsymbol{r}}}\left[\tilde{G}_{\mathrm{loc}}^{(0)-1} \tilde{G}_{\mathrm{nonloc}}^{(0)} \tilde{G}_{\mathrm{loc}}^{(0)-1}\right]_{\tilde{\boldsymbol{r}}, \tilde{\boldsymbol{r}}^{\prime}} \theta_{\tilde{\boldsymbol{r}}^{\prime}}
$$

where $H_{\mathrm{eff}}^{(1)}\left(\theta_{\tilde{\boldsymbol{r}}}, \theta_{\tilde{\boldsymbol{r}}+z}\right)$ is given by Eq. (3.9).

Since this term is dominant at low impurity concentration, it is again reasonable to restrict the angular configurations to the subspace given by

$$
\theta_{\tilde{\boldsymbol{r}}}=-\theta_{\tilde{\boldsymbol{r}}+z}=\frac{\Delta \theta_{\tilde{\boldsymbol{r}}}}{2} \Longrightarrow \theta_{\tilde{\boldsymbol{r}}}+\theta_{\tilde{\boldsymbol{r}}+z}=0, \quad \theta_{\tilde{\boldsymbol{r}}}-\theta_{\tilde{\boldsymbol{r}}+z}=\Delta \theta_{\tilde{\boldsymbol{r}}}
$$

as in the single impurity-bond problem of Eq. (3.9). This leads to the effective restricted Hamiltonian [compare with Eq. (3.13)]

$$
\begin{aligned}
H_{\mathrm{eff} / \mathrm{res}}\left(\left\{\Delta \theta_{\tilde{\boldsymbol{r}}}\right\}\right) & :=\sum_{\tilde{\boldsymbol{r}} \in \mathcal{L}} H_{\mathrm{eff} / \mathrm{res}}^{(1)}\left(\Delta \theta_{\tilde{\boldsymbol{r}}}\right)-\frac{1}{2} \sum_{\tilde{\boldsymbol{r}} \neq \tilde{\boldsymbol{r}}^{\prime} \in \mathcal{L}} \frac{\Delta \theta_{\tilde{\boldsymbol{r}}}}{2\left(G_{\mathbf{0}}^{(0)}-G_{z}^{(0)}\right)}\left(2 G_{\tilde{\boldsymbol{r}}, \tilde{\boldsymbol{r}}^{\prime}}-G_{\tilde{\boldsymbol{r}}+z, \tilde{\boldsymbol{r}}^{\prime}}-G_{\tilde{\boldsymbol{r}}, \tilde{\boldsymbol{r}}^{\prime}+z}\right) \frac{\Delta \theta_{\tilde{\boldsymbol{r}}^{\prime}}}{2\left(G_{\mathbf{0}}^{(0)}-G_{z}^{(0)}\right)} \\
& =\sum_{\tilde{\boldsymbol{r}} \in \mathcal{L}} H_{\mathrm{eff} / \mathrm{res}}^{(1)}\left(\Delta \theta_{\tilde{\boldsymbol{r}}}\right)-\frac{1}{2\left(\Gamma_{\mathbf{0}}^{(0)}\right)^{2}} \sum_{\tilde{\boldsymbol{r}} \neq \tilde{\boldsymbol{r}}^{\prime} \in \mathcal{L}} \Delta \theta_{\tilde{\boldsymbol{r}}} \Gamma_{\tilde{\boldsymbol{r}}-\tilde{\boldsymbol{r}}^{\prime}}^{(0)} \Delta \theta_{\tilde{\boldsymbol{r}}^{\prime}},
\end{aligned}
$$

where the interaction $\Gamma_{\tilde{\boldsymbol{r}}-\tilde{\boldsymbol{r}}^{\prime}}^{(0)}$ is seen to be mediated by the combination of Green's functions introduced in Eq. (2.14a) that scales like an antidipolar interaction at long distances. As it should be, the effective Hamiltonian is invariant under both the global rotation $\theta_{\tilde{r}} \mapsto \theta_{\tilde{r}}+\Theta, \Theta \in[0,2 \pi$ [, and the global Ising symmetry $\theta_{\tilde{\boldsymbol{r}}} \mapsto-\theta_{\tilde{\boldsymbol{r}}}$ for all $\tilde{\boldsymbol{r}} \in \mathcal{L}$.
It remains to minimize $H_{\mathrm{eff} / \mathrm{res}}\left(\left\{\Delta \theta_{\tilde{r}}\right\}\right)$ with respect to the canting angles $\Delta \theta_{\tilde{\boldsymbol{r}}}$ on the impurity bonds. The corresponding saddle-point equation reads

$$
\Delta \theta_{\tilde{\boldsymbol{r}}}=\frac{\left|J_{\mathrm{imp}}\right|}{J_{\mathrm{c}}} \sin \Delta \theta_{\tilde{\boldsymbol{r}}}+\Xi_{\tilde{\boldsymbol{r}}}
$$


where

$$
\Xi_{\tilde{\boldsymbol{r}}}:=\frac{1}{J_{\mathrm{c}}} \sum_{\tilde{\boldsymbol{r}}^{\prime} \in \mathcal{L} \backslash\{\tilde{r}\}} \frac{\Gamma_{\tilde{r} \boldsymbol{r}}^{(0)} \tilde{r}^{\prime}}{\left(\Gamma_{\mathbf{0}}^{(0)}\right)^{2}} \Delta \theta_{\tilde{\boldsymbol{r}}^{\prime}} .
$$

As before, the ferromagnetic state (3.14) is a solution of Eqs. (3.27a) and (3.27b), but it becomes unstable for sufficiently large $\left|J_{\text {imp }}\right|$.

The term $\Xi_{\tilde{r}}$ is expected to be dominated by the closestneighbor bonds, since the sum is over a set of decreasing terms, which, for a valid saddle point, contribute with alternating signs. Using Eqs. (2.15) and (3.27b), one expects that $\Xi_{\tilde{r}}$ scales as $n_{\text {imp. }}$. In that case, the condition

$$
\frac{\left|J_{\text {imp }}\right|}{J_{\mathrm{c}}} \gg \Xi_{\tilde{r}}
$$

will be met for sufficiently large values of $J_{\text {imp }}$ and sufficiently small values of $n_{\text {imp }}$. Under the condition (3.28), local minima of the Hamiltonian (3.1a), i.e., solutions to Eq. (3.27), exist, which locally look like the solution for a single antiferromagnetic impurity bond.

In the dilute impurity regime, a low-energy state will have a canting of angles across the impurities bonds close to the single impurity case, i.e.,

$$
\Delta \theta_{\tilde{\boldsymbol{r}}} \approx \sigma_{\tilde{\boldsymbol{r}}} \Delta \theta
$$

Inserting this ansatz into the effective Hamiltonian (3.26), we obtain the effective Ising model

$$
\begin{aligned}
H_{\mathcal{L}}\left[\sigma_{\tilde{r}}\right]:= & E_{\mathrm{FM}}+E(\Delta \theta)|\mathcal{L}| \\
& -\frac{1}{2}\left(\frac{\Delta \theta}{\Gamma_{\mathbf{0}}^{(0)}}\right)^{2} \sum_{\tilde{r} \neq \tilde{r}^{\prime} \in \mathcal{L}} \sigma_{\tilde{r}} \Gamma_{\tilde{\boldsymbol{r}}-\tilde{r}^{\prime}}^{(0)} \sigma_{\tilde{r}^{\prime}} .
\end{aligned}
$$

This effective Ising model is invariant under the global Ising symmetry $\sigma_{\tilde{r}} \mapsto-\sigma_{\tilde{\boldsymbol{r}}}$ for all $\tilde{\boldsymbol{r}} \in \mathcal{L}$. Equation $(3.29 \mathrm{~b})$ is the main result of Sec. III B.

The interaction $\Gamma_{\tilde{r}}^{(0)}$ between a pair of Ising variables a distance $\tilde{\boldsymbol{r}}$ apart that enters on the right-hand side of Eq. (3.29b) was derived in Eq. (2.15). Its asymptotic behavior is that of Ising dipoles ( $\sigma_{\tilde{r}}$ oriented along the direction $z$ ), albeit with the opposite sign as compared to the usual dipolar interaction. In the dilute impurity limit, the sign of the two-body interaction $\Gamma_{r}^{(0)}$ depends on the relative position $\tilde{\boldsymbol{r}}$ (see Fig. 4). It is ferromagnetic when

$$
\tilde{x}^{2}+\tilde{y}^{2}>\frac{2 J_{\|}}{J_{\perp}} \tilde{z}^{2}
$$

vanishing on the conical surface

$$
\tilde{x}^{2}+\tilde{y}^{2}=\frac{2 J_{\|}}{J_{\perp}} \tilde{z}^{2}
$$

and antiferromagnetic when

$$
\tilde{x}^{2}+\tilde{y}^{2}<\frac{2 J_{\|}}{J_{\perp}} \tilde{z}^{2}
$$

\section{Boundary conditions along the $z$ axis}

So far, we have considered the ferromagnetic state, $\theta_{\boldsymbol{r}}=0$ for all $\boldsymbol{r} \in \Lambda$, of $H_{0}$ defined in Eq. (2.4) and performed a

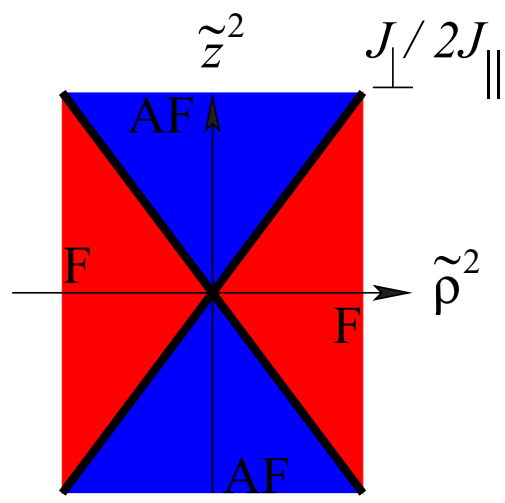

FIG. 4. Sign of the (anti)dipolar interaction $\Gamma_{\tilde{r}}^{(0)}$ [Eq. (2.15)] between cantings, in the $\tilde{\rho}^{2}-\tilde{z}^{2}$ plane (with $\tilde{\rho}^{2} \equiv \tilde{x}^{2}+\tilde{y}^{2}$ ). Along the $z$ axis the interactions are antiferromagnetic, while for separation along the $x-y$ plane they are ferromagnetic. The sign change occurs on the cone described by $J_{\perp} \tilde{\rho}^{2}=2 J_{\|} \tilde{z}^{2}$. In the quasi-one-dimensional limit $J_{\|} / J_{\perp} \rightarrow 0$ the interaction $\Gamma_{\tilde{r}}^{(0)}$ is ferromagnetic for any $\tilde{\rho}^{2}>0$. In the quasi-two-dimensional limit $J_{\perp} / J_{\|} \rightarrow 0, \Gamma_{\tilde{r}}^{(0)}$ is antiferromagnetic for any $\tilde{z}^{2}>0$.

spin-wave expansion about it, imposing periodic boundary conditions. Imposing periodic boundary conditions precludes an instability to a spiral state within our self-consistent approximation. To overcome this technical limitation, we allow for a linear growth of

$$
\theta_{\boldsymbol{r}}=\phi_{\boldsymbol{r}}+Q(\boldsymbol{r} \cdot \boldsymbol{z}),
$$

along the $z$ direction. Here the global degree of freedom $Q \in[-\pi, \pi$ [ describes a constant twist rate, while the local degrees of freedom $\phi_{r}$ obey periodic boundary conditions. In finite systems, the twist rate $Q$ along the $z$ direction should be an integer multiple of $2 \pi / L_{z}$ if we impose periodic boundary conditions on the original spins $\hat{\boldsymbol{S}}_{\boldsymbol{r}}=\cos \theta_{r} \hat{\boldsymbol{x}}+\sin \theta_{\boldsymbol{r}} \hat{\boldsymbol{y}}$, but this discrete constraint is irrelevant in the thermodynamic limit. With the change of variables (3.31), the spin-wave approximation (3.1a) becomes

$$
\begin{aligned}
H_{\mathcal{L}} \approx & E_{\mathrm{FM}}+\frac{1}{2} \sum_{r, r^{\prime} \in \Lambda} \phi_{r} D_{r-r^{\prime}}^{(0)} \phi_{r^{\prime}}+\frac{J_{\perp} Q^{2}}{2}|\Lambda| \\
& -\sum_{\tilde{r} \in \mathcal{L}}\left(\frac{J_{\perp}}{2}\left(\Delta \phi_{\tilde{r}}-Q\right)^{2}-\left|J_{\mathrm{imp}}\right| \cos \left(\Delta \phi_{\tilde{\boldsymbol{r}}}-Q\right)\right),
\end{aligned}
$$

where we recall that $|\Lambda|$ is the number of sites in the host cubic lattice $\Lambda$ and we again define by

$$
\Delta \phi_{\tilde{r}}:=\phi_{\tilde{r}}-\phi_{\tilde{r}+z}
$$

the twist of $\phi$ across the impurity bond labeled by $\tilde{\boldsymbol{r}}$.

For a low impurity concentration $0<n_{\text {imp }} \ll 1$, we assume and will verify a posteriori that

$$
|Q| \sim n_{\text {imp }} \ll \Delta \theta,
$$

where $\Delta \theta \geqslant 0$ is the modulus of the canting angle across an isolated impurity bond. The leading effect of the emerging spiral order will appear at $O\left(n_{\text {imp }}^{2}\right)$ in the energy per spin. We therefore expand the impurity bond terms in Eq. (3.32a), 
i.e., the second line on the right-hand side of Eq. (3.32a), up to linear order in $Q$. In this approximation, the saddle-point values for $\Delta \phi_{\tilde{r}}$ are therefore again given by

$$
\Delta \phi_{\tilde{r}} \approx \sigma_{\tilde{r}} \Delta \theta
$$

up to corrections of $O(Q)$. We can neglect those since they lead to corrections to the energy per spin of $O\left(n_{\mathrm{imp}}^{3}\right)$. The angles of spins that do not belong to impurity bonds are again given by Eq. (3.27), with $\Delta \phi_{\tilde{\boldsymbol{r}}}$ replacing $\Delta \theta_{\tilde{\boldsymbol{r}}}$. However, minimizing over the twist rate $Q$ after linearization with respect to $Q$ of the second line on the right-hand side of Eq. (3.32a) yields the nontrivial saddle-point value

$$
\begin{aligned}
Q & =-\frac{1}{|\Lambda|} \sum_{\tilde{\boldsymbol{r}} \in \mathcal{L}}\left(\frac{\left|J_{\text {imp }}\right|}{J_{\perp}} \sin \left(\Delta \phi_{\tilde{\boldsymbol{r}}}\right)+\Delta \phi_{\tilde{\boldsymbol{r}}}\right) \\
& =-\Delta \theta \frac{J_{\mathrm{c}}+J_{\perp}}{J_{\perp}} \frac{1}{|\Lambda|} \sum_{\tilde{\boldsymbol{r}} \in \mathcal{L}} \sigma_{\tilde{\boldsymbol{r}}},
\end{aligned}
$$

where we have used the saddle-point equation (3.15) to reach the second equality. A spontaneous net winding $(Q \neq 0)$ of the spins along the $z$ direction thus occurs for canting configurations $\left\{\sigma_{\tilde{r}}\right\}$ with a net bias. An Ising configuration $\left\{\sigma_{\tilde{r}}\right\}$ with a net uniform magnetization thus corresponds to a spiral state for the $X Y$ spins. Note that the wave vector $Q$ of the spiral is proportional to the magnetization density of the Ising variables.

We can now verify a posteriori the validity of the assumption (3.33). The maximal value of $|Q|$ is given by

$$
|Q|_{\max }=n_{\mathrm{imp}} \frac{J_{\mathrm{c}}+J_{\perp}}{J_{\perp}} \Delta \theta .
$$

Thus, for

$$
n_{\text {imp }} \ll \frac{J_{\perp}}{J_{\mathrm{c}}+J_{\perp}},
$$

our assumption is certainly self-consistent. In the opposite regime, as $n_{\text {imp }}$ approaches 1 from below, the interaction between the planes starts to be dominated by the impurity bonds, which may induce an entirely different ground state with no spiral order.

Injecting the saddle-point value of $Q$ [Eq. (3.35)] into Eq. (3.32) and expressing the energy as a function of the Ising variables $\sigma_{\tilde{r}}$ leads to the same effective Hamiltonian as in Eq. (3.29b), except for an additional term $-\frac{J_{\perp} Q^{2}}{2}|\Lambda|$, which expresses the lowering of the total energy due to the coupling of the canting pattern to the spiral order,

$$
\begin{aligned}
H_{\mathcal{L}}\left[\sigma_{\tilde{\boldsymbol{r}}}\right]:= & E_{\mathrm{FM}}+E(\Delta \theta)|\mathcal{L}| \\
& -\frac{\gamma}{2} \sum_{\tilde{\boldsymbol{r}} \neq \tilde{\boldsymbol{r}}^{\prime} \in \mathcal{L}} \sigma_{\tilde{\boldsymbol{r}}} \Gamma_{\tilde{\boldsymbol{r}}-\tilde{\boldsymbol{r}}^{\prime}}^{(0)} \sigma_{\tilde{\boldsymbol{r}}^{\prime}}-\frac{J_{\perp} Q^{2}}{2}|\Lambda| \\
= & E_{\mathrm{FM}}+E(\Delta \theta)|\mathcal{L}| \\
& -\frac{1}{2} \sum_{\tilde{\boldsymbol{r}} \neq \tilde{\boldsymbol{r}}^{\prime} \in \mathcal{L}} \sigma_{\tilde{\boldsymbol{r}}} J_{\tilde{\boldsymbol{r}}-\tilde{\boldsymbol{r}}^{\prime}}^{(\mathrm{I})} \sigma_{\tilde{\boldsymbol{r}}^{\prime}}-\frac{\gamma n_{\mathrm{imp}}}{2 J_{\perp}},
\end{aligned}
$$

where we have introduced the constant

$$
\gamma:=(\Delta \theta)^{2}\left(J_{\mathrm{c}}+J_{\perp}\right)^{2}=\left(\frac{\Delta \theta}{\Gamma_{\mathbf{0}}^{(0)}}\right)^{2}
$$

and the effective Ising interaction

$$
J_{\tilde{\boldsymbol{r}}-\tilde{\boldsymbol{r}}^{\prime}}^{(\mathrm{I})}:=\gamma\left(\Gamma_{\tilde{\boldsymbol{r}}-\tilde{\boldsymbol{r}}^{\prime}}^{(0)}+\frac{1}{J_{\perp}|\Lambda|}\right) .
$$

Note that the last term in Eq. (3.38a) is nonextensive and thus irrelevant in the thermodynamic limit.

There are two additive contributions to the Ising exchange coupling $J_{\tilde{\boldsymbol{r}}-\tilde{\boldsymbol{r}}^{\prime}}^{(\mathrm{I})}$. The contribution $\Gamma_{\tilde{\boldsymbol{r}}-\tilde{\boldsymbol{r}}^{\prime}}^{(0)}$ represents an antidipolar two-body interaction between the effective Ising degrees of freedom $\sigma_{\tilde{r}}$ associated with the dilute antiferromagnetic bonds. This interaction is long ranged and frustrated, owing to the indefinite sign of the kernel $\Gamma_{\tilde{r}-\tilde{r}^{\prime}}^{(0)}$. The coupling of the canting pattern to the spiral order instead favors a net (ferromagnetic) bias of the cantings $\sigma_{\tilde{r}}$ and contributes an allto-all interaction of strength $\frac{1}{J_{\perp}|\Lambda|}$, proportional to the inverse volume.

According to the saddle-point equation (3.27a), a uniform magnetization of the Ising spins $\sigma_{\tilde{r}}$ favors a spiral state with a nonvanishing $Q$ for the original $\mathrm{O}(2)$ spin degrees of freedom. Hence, if the ground state of Eq. (3.38) supports a nonvanishing magnetization $\sigma_{\tilde{r}}$, the frustration induced by the dilute impurity bonds turns the pristine ferromagnetic order of the impurity-free ground state into spiral order. If instead the ground state supports no net bias of the Ising spins $\sigma_{\tilde{r}}$, a state with $Q=0$ is favored, and thus no net winding of the $\mathrm{O}(2)$ spins is induced. An example of a possible resulting ground state is a fanlike magnetic state where the Ising spins order in a layered antiferromagnetic pattern. This translates into a pattern of the original $\mathrm{O}(2)$ spins ordering essentially ferromagnetically, but with orientations that alternate slightly between successive layers.

The Ising Hamiltonian with antidipolar coupling (3.38) is structurally very similar to Ising systems with standard dipolar couplings, as are realized, e.g., in rare-earth compounds with strongly localized magnetic moments, such as $\mathrm{LiHo}_{x} \mathrm{Y}_{1-x} \mathrm{~F}_{4}$ at moderate to low dilution $x$ [27]. It has been theoretically and experimentally well established that such random dipolar Ising systems exhibit a glass transition towards an amorphous magnetic order at low temperature [20,28]. It may thus come as a surprise that in our case we find that a change of sign of the dipolar term, in conjunction with the additional term arising from the coupling to the spiral, suffices to induce ferromagnetic Ising order, in spite of the positional randomness of the Ising spins. This difference is presumably largely due to the additional mean-field-like interaction mediated by the formation of the spiral, which stabilizes the ferromagnetic phase. That type of interaction is absent in systems of elementary magnetic dipoles, which therefore fall much more easily into a glassy phase.

\section{SUPERLATTICES OF IMPURITY BONDS}

As Eq. (3.38) involves long-range two-body interactions whose sign depends on the relative positions of the impurity bonds, the ground state cannot be found explicitly for an arbitrary choice of $\mathcal{L}$, so in general one has to resort to numerical methods or to approximate treatments. However, if the set of impurity bonds $\mathcal{L}$ realizes certain Bravais superlattices, it is possible to establish a sufficient condition for the ground state of the effective Ising Hamiltonian (3.38) to be 
ferromagnetic and thus for the ground-state spin configuration of the Hamiltonian (3.1a) to sustain spiral order.

\section{A. Analytical considerations}

We consider the case when the subset $\mathcal{L}$ of the cubic host lattice $\Lambda$ forms a Bravais lattice with the basis vectors $\boldsymbol{A}, \boldsymbol{B}$, and $\boldsymbol{C}$ given by three independent linear combinations with integer-valued coefficients of $\boldsymbol{a} \equiv(1,0,0)^{\top}, \boldsymbol{b} \equiv(0,1,0)^{\top}$, and $c \equiv(0,0,1)^{\top}$. The concentration of the impurity bonds is

$$
n_{\mathrm{imp}} \equiv \frac{1}{|\boldsymbol{A} \cdot(\boldsymbol{B} \wedge \boldsymbol{C})|}
$$

In reciprocal space, the superlattice $\mathcal{L}$ defines a small first Brillouin zone $\operatorname{BZ}(\mathcal{L})$, which is $1 / n_{\text {imp }}$ times smaller than the first Brillouin zone $\operatorname{BZ}(\Lambda)$ of the cubic host lattice $\Lambda$.

For the $|\mathcal{L}|$ Ising degrees of freedom $\sigma_{\tilde{r}}$ associated with impurity bonds anchored at $\tilde{\boldsymbol{r}} \in \mathcal{L}$, we use the Fourier representation

$$
\sigma_{\tilde{\boldsymbol{r}}}=\frac{1}{|\mathcal{L}|} \sum_{\boldsymbol{q} \in \mathrm{BZ}(\mathcal{L})} e^{i \boldsymbol{q} \cdot \tilde{r}} \sigma_{\boldsymbol{q}}
$$

where $\mathrm{BZ}(\mathcal{L})$ is the first Brillouin zone associated with the lattice $\mathcal{L}$. For any $\boldsymbol{p} \in \mathbb{R}^{3}$, we will make use of the identity

$$
\frac{1}{|\mathcal{L}|} \sum_{\tilde{\boldsymbol{r}} \in \mathcal{L}} e^{i \boldsymbol{p} \cdot \tilde{\boldsymbol{r}}}=\sum_{\mathbf{G} \in \mathcal{L}^{\star}} \delta_{\boldsymbol{p}, \mathbf{G}}
$$

$$
\phi_{\boldsymbol{r}}^{\min }:=n_{\mathrm{imp}} \sqrt{\gamma} \sum_{\left\{\boldsymbol{k} \in \mathrm{BZ}(\Lambda) \mid \boldsymbol{k}-\boldsymbol{q}_{\min } \in \mathcal{L}^{\star} \backslash\{\mathbf{0}\}\right\}} \frac{\left(1-e^{-i k_{z}}\right) e^{i \boldsymbol{k} \cdot \boldsymbol{r}}}{2 J_{\|}\left(2-\cos k_{x}-\cos k_{y}\right)+2 J_{\perp}\left(1-\cos k_{z}\right)},
$$

with $\gamma$ defined by Eq. (3.38b) and

$$
Q^{\min }:=-\frac{\sqrt{\gamma}}{J_{\perp}} n_{\mathrm{imp}} \delta_{\boldsymbol{q}_{\min }, \mathbf{0}} .
$$

Examples of $\boldsymbol{q}_{\min }$ for which Eq. (4.5) holds are

$$
\boldsymbol{q}_{\min }=\mathbf{0}
$$

and

$$
\boldsymbol{q}_{\text {min }} \in\left\{ \pm \frac{1}{2} \boldsymbol{A}^{\star}, \pm \frac{1}{2} \boldsymbol{B}^{\star}, \pm \frac{1}{2} \boldsymbol{C}^{\star}\right\}
$$

where $\boldsymbol{A}^{\star}, \boldsymbol{B}^{\star}$, and $\boldsymbol{C}^{\star}$ are the basis vectors of the reciprocal lattice $\mathcal{L}^{\star}$. In the former case (4.7a), $Q^{\text {min }} \neq 0$ and the ground state is an $\mathrm{O}(2)$ magnetic spiral, while in the latter case (4.7b), $Q^{\min }=0$ and thus there is no spiral.

\section{B. Comparison between analytical and numerical results for superlattices}

To illustrate that the effective Ising Hamiltonian (3.38) captures the low-energy physics of the microscopic Hamiltonian (2.1), we consider several superlattices $\mathcal{L}$ of impurity bonds and compare their microscopic ground state to the ground where $\mathcal{L}^{\star}$ denotes the reciprocal lattice of $\mathcal{L}$. The effective Ising Hamiltonian (3.38) can now be written in reciprocal space as

$$
H_{\mathcal{L}}=E_{\mathrm{FM}}+E(\Delta \theta)|\mathcal{L}|+\frac{\gamma}{2|\mathcal{L}|} \sum_{\boldsymbol{q} \in \mathrm{BZ}(\mathcal{L})} \Upsilon_{\boldsymbol{q}} \sigma_{+\boldsymbol{q}} \sigma_{-\boldsymbol{q}},
$$

where $\gamma$ is defined by Eq. (3.38b) and

$$
\Upsilon_{\boldsymbol{q}}:=-\sum_{\tilde{\boldsymbol{r}} \in \mathcal{L} \backslash\{\mathbf{0}\}} e^{-i \boldsymbol{q} \cdot \tilde{\boldsymbol{r}}} \Gamma_{\tilde{\boldsymbol{r}}}^{(0)}-\frac{n_{\mathrm{imp}}}{J_{\perp}} \delta_{\boldsymbol{q}, \mathbf{0}} .
$$

Using Eq. (4.3), we obtain

$$
\Upsilon_{\boldsymbol{q}}=-n_{\mathrm{imp}} \sum_{\substack{\mathbf{G} \in \mathcal{L}^{\star} \\ \boldsymbol{q}+\mathbf{G} \in \mathrm{BZ}(\Lambda)}} \Gamma_{\boldsymbol{q}+\mathbf{G}}^{(0)}+\Gamma_{\tilde{\boldsymbol{r}}=\mathbf{0}}^{(0)}-\frac{n_{\mathrm{imp}}}{J_{\perp}} \delta_{\boldsymbol{q}, \mathbf{0}} .
$$

For a generic choice of the Bravais lattice $\mathcal{L}$ and of the couplings $J_{\perp}, J_{\|}$, and $J_{\text {imp }}$, the ground state of the Ising Hamiltonian (4.4) cannot be found in closed form. However, an analytical solution is available in certain cases. For instance, if over the reduced Brillouin zone $\mathrm{BZ}(\mathcal{L})$ the kernel $\Upsilon_{q}$ assumes its global minimum at a unique momentum $\boldsymbol{q}_{\min }$ such that for all $\tilde{\boldsymbol{r}} \in \mathcal{L}$,

$$
\sigma_{\tilde{\boldsymbol{r}}}^{\min }=e^{i \boldsymbol{q}_{\min } \cdot \tilde{\boldsymbol{r}}}= \pm 1,
$$

the ground state of the Ising Hamiltonian is then given by the configuration described by Eq. (4.5). Inserting Eq. (4.5) into Eqs. (3.31), (3.34), and (3.35) and using the Fourier representation (2.14a) and (2.14b), one finds

$$
\theta_{\boldsymbol{r}}^{\min }=\phi_{\boldsymbol{r}}^{\min }+Q^{\min }(\boldsymbol{r} \cdot \boldsymbol{z}),
$$

where

state of the effective Ising Hamiltonian (4.4). Instead of directly studying the ground state of the microscopic Hamiltonian (2.1), we actually study the microscopic Hamiltonian

$$
\begin{aligned}
H_{\text {Heis }}:= & -\frac{1}{2} \sum_{\boldsymbol{r}, \boldsymbol{r}^{\prime} \in \Lambda} J_{\boldsymbol{r}, \boldsymbol{r}^{\prime}}^{(0)} \boldsymbol{S}_{\boldsymbol{r}} \cdot \boldsymbol{S}_{\boldsymbol{r}^{\prime}} \\
& +\left(\left|J_{\mathrm{imp}}\right|+J_{\perp}\right) \sum_{\tilde{\boldsymbol{r}} \in \mathcal{L}} \boldsymbol{S}_{\tilde{\boldsymbol{r}}} \cdot \boldsymbol{S}_{\tilde{\boldsymbol{r}}+z}+\Delta \sum_{\boldsymbol{r}}\left(S_{\boldsymbol{r}}^{z}\right)^{2},
\end{aligned}
$$

which is closer to experimental realizations. Here we have replaced the classical $X Y$ spins from Eq. (2.1) with classical Heisenberg spins $\boldsymbol{S}_{\boldsymbol{r}}$ (being unit vectors in $\mathbb{R}^{3}$ ). A single-ion anisotropy $\Delta>0$ penalizes a spin orientation along the $z$ axis. We apply open boundary conditions along all principal directions of the cubic lattice. To approximately find the ground state, we perform parallel tempering Monte Carlo (MC) simulations. We use 140 temperatures $T_{i}$ with a constant ratio $T_{i+1} / T_{i}$, covering a range from $T \leqslant 4 \times 10^{-3} J_{\|}$up to temperatures well in the paramagnetic phase. The ground state is obtained by keeping track of the minimal-energy state visited during the Monte Carlo evolution for the lowest 


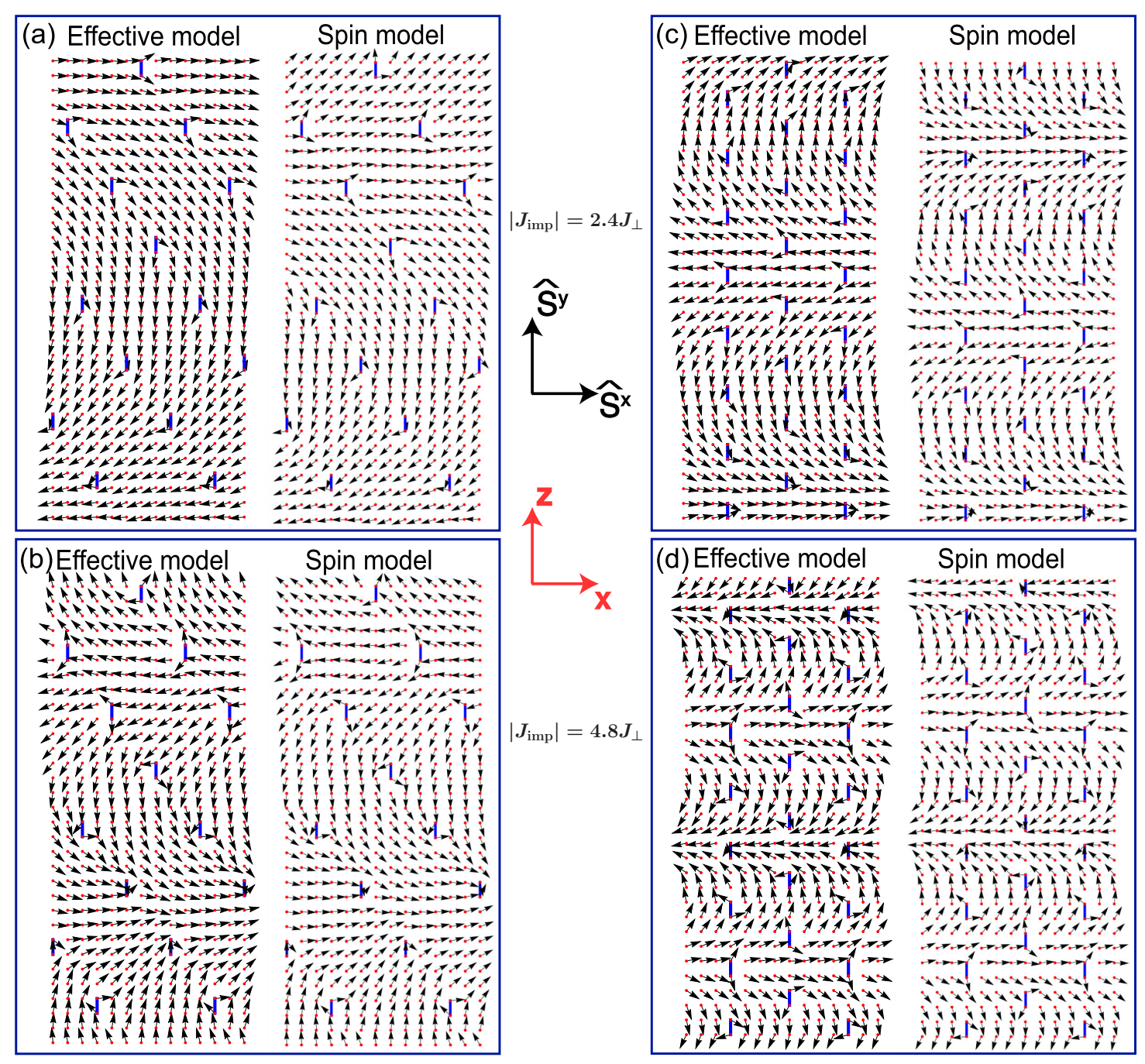

FIG. 5. Spin configurations of the ground state in an $x$-z plane of the cubic host lattice $\Lambda$. Black arrows represent the $\mathrm{O}(2)(X Y)$ spins defined in Eq. (2.3) (black reference frame). Red dots represent the sites in a cross section of the cubic host lattice $\Lambda$ (red reference frame). Blue lines represent the impurity bonds. Results are obtained for $J_{\|} / J_{\perp}=1$. Each panel (a)-(d) shows a comparison between approximate analytical results based on the effective model, and MC results from the full spin model. In panels (a) and (b) a superlattice with the basis $\boldsymbol{A}=(5,3,2)^{\top}, \boldsymbol{B}=(3,4,4)^{\top}$, and $\boldsymbol{C}=(4,5,2)^{\top}$ is chosen, in panels (c) and (d) the superlattice has the basis $\boldsymbol{A}=(3,3,2)^{\top}, \boldsymbol{B}=(0,4,2)^{\top}$, and $\boldsymbol{C}=(4,0,2)^{\top}$. The impurity strengths are $\left|J_{\text {imp }} / J_{\perp}\right|=2.4\left((\mathrm{a})\right.$ and (c)) and $\left|J_{\text {imp }} / J_{\perp}\right|=4.8((\mathrm{~b})$ and (d)). In all cases the effective Ising Hamiltonian (4.4) correctly predicts a spiral state. However, the accuracy of the predicted value of $Q$ significantly improves with increasing $\left|J_{\text {imp }} / J_{\perp}\right|$ away from the critical value of 2 , where canting sets in. This can be seen by comparing the relative orientations of the spins in the lattice corners.

temperature. The single-ion anisotropy is restricted to $0<$ $\Delta \leqslant 0.02\left|J_{\|}\right|$. For $T \ll \Delta$, the low-energy states are essentially coplanar with spins lying in the $x-y$ plane. At $T=0$ the ground state of the $X Y$ Hamiltonian (2.1) is identical to that of the anisotropic Heisenberg Hamiltonian (4.8). The typical size of the cubic host lattice $\Lambda$ used in the MC simulations is $14 \times 14 \times 32$ lattice spacings.

To obtain the corresponding effective model in terms of cantings, we take the following steps. First, we verify that the exchange couplings allow for a nontrivial solution $\Delta \theta \neq$ 0 of Eq. (3.15). Second, we solve for the ground state of the effective Ising Hamiltonian (4.4). To this end we minimize the function $\Upsilon_{q}$ in Eq. (4.4c) with respect to $q$. If the absolute minimum in the Brillouin zone $\operatorname{BZ}(\mathcal{L})$ occurs at $\boldsymbol{q}=0$, we predict a spiral ground state $(Q \neq 0)$. If the absolute minimum occurs at $\boldsymbol{q}_{\min }=\boldsymbol{C}^{\star} / 2$, a ground state with $Q=0$ is predicted. If instead $\boldsymbol{q}_{\text {min }}$ does not satisfy Eq. (4.5), the ground state has more than one Fourier component and we would need to solve the effective Ising model numerically. Third, from the Ising ground state of the Hamiltonian (4.4), the microscopic pattern (2.3) of the $\mathrm{O}(2)$ spins is finally obtained from Eq. (4.6), using the value of $\Delta \theta$ obtained from solving Eq. (3.15).

The effective Ising Hamiltonian (4.4) is found to be very accurate once the impurity coupling $\left|J_{\text {imp }}\right|$ sufficiently exceeds the critical value $J_{\mathrm{c}}$. This is illustrated by Fig. 5. Its four panels compare the approximate ground state obtained via the effective Ising Hamiltonian (4.4a) (shown on the left) with the ground state of the Hamiltonian (4.8) obtained via MC 
(a)

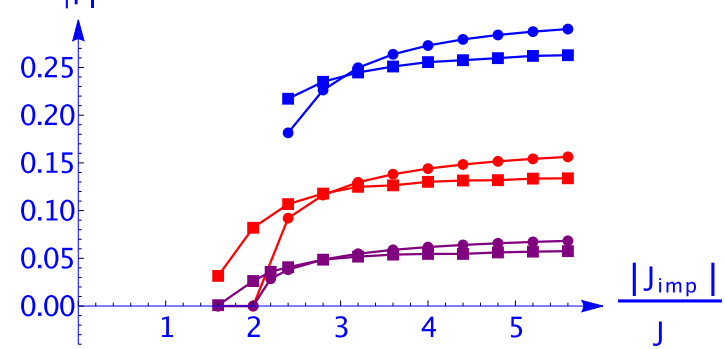

(b) $\mid \mathrm{P}$

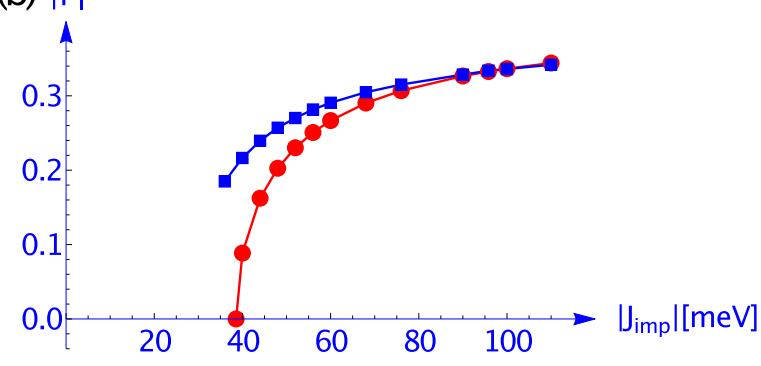

FIG. 6. Comparison between the approximate analytical (circles) and numerical (squares) values of the spiral order parameter $|P|$ [cf. Eq. (4.11)] for $X Y$ spins on a cubic lattice with $L_{x}=14, L_{y}=14$, and $L_{z}=32$. The impurity bonds form a superlattice. (a) Results for the isotropic case $J_{\|} / J_{\perp}=1$ for three superlattices with the following basis vectors: $\boldsymbol{A}=(3,3,2)^{\top}, \boldsymbol{B}=(0,4,2)^{\top}$, and $\boldsymbol{C}=(4,0,2)^{\top}$, with $n_{\text {imp }}=1 / 16$ (blue); $\boldsymbol{A}=(5,3,2)^{\top}, \boldsymbol{B}=(3,4,4)^{\top}$, and $\boldsymbol{C}=(4,5,2)^{\top}$, with $n_{\text {imp }}=1 / 32$ (red); and $A=(4,3,0)^{\top}, B=(0,4,3)^{\top}$, and $C=$ $(5,0,2)^{\top}$, with $n_{\text {imp }}=1 / 77$ (purple). Note that the magnitude $|P|$ of the spiral order parameter $P$ increases approximately linearly with $n_{\text {imp. }}$. (b) Dependence of $|P|$ on $\left|J_{\text {imp }}\right|$, for the parameters of $\mathrm{YBaCuFeO}_{5}$ [cf. Eq. (4.12)], and a superlattice of density $n_{\mathrm{imp}}=1 / 56$, with the basis $\boldsymbol{A}=(4,3,0)^{\top}, \boldsymbol{B}=(0,4,2)^{\top}$, and $\boldsymbol{C}=(4,0,2)^{\top}$

simulation (shown on the right). This is done for two strengths of impurity couplings and two different superlattices. We choose parameters such that both methods yield a spiral state, with $\boldsymbol{q}_{\min }=0$ minimizing the kernel $\Upsilon_{\boldsymbol{q}}$. No coupling anisotropy $\left(J_{\|} / J_{\perp}=1\right)$ was assumed in all these cases. Figures 5(a) and 5(b) correspond to the same superlattice, but different impurity strengths $\left|J_{\text {imp }}\right| / J_{\perp}=2.4$ and 4.8 , respectively. A spiral ground state is correctly predicted in both cases. However, while the canting angle $\Delta \theta$ at the impurity bonds and especially the spiral wave vector $Q$ are rather accurately predicted for strong impurity couplings $\left|J_{\text {imp }}\right| / J_{\perp}=$ 4.8 , they are underestimated by the effective theory when the impurity coupling $\left|J_{\mathrm{imp}}\right| / J_{\perp}=2.4$ comes relatively close to the threshold strength $J_{\mathrm{c}} / J_{\perp}=2$ [cf. Eq. (3.13b)]. The agreement between the analytical approximation and the MC simulations improves with increasing $\left|J_{\text {imp }}\right| / J_{\perp}$. This agrees with what one expects from the considerations of Sec. III B. Indeed, assuming that all canting angles take the same value $\Delta \theta_{\tilde{r}}=\Delta \theta$ and assuming a superlattice of impurities, Eq. (3.27) takes the form

$$
\Delta \theta=\frac{\left|J_{\text {imp }}\right|}{J_{\mathrm{c}}-\left(\Gamma_{\mathbf{0}}^{(0)}\right)^{-2} \sum_{\tilde{\boldsymbol{r}} \in \mathcal{L} \backslash\{\mathbf{0}\}} \Gamma_{\tilde{\boldsymbol{r}}}^{(0)}} \sin \Delta \theta .
$$

As compared to the saddle-point equation for a single impurity [Eq. (3.15)], the denominator $J_{\mathrm{c}}$ is shifted by the small correction

$$
\left(\Gamma_{\mathbf{0}}^{(0)}\right)^{-2} \sum_{\tilde{\boldsymbol{r}} \in \mathcal{L} \backslash\{\mathbf{0}\}} \Gamma_{\tilde{\boldsymbol{r}}}^{(0)} \sim n_{\mathrm{imp}} O\left(J_{\perp}, J_{\|}\right) \ll J_{\mathrm{c}} .
$$

When $\left|J_{\text {imp }}\right| / J_{c}$ is large, this renormalization has little effect on the solution of the saddle-point equation $\Delta \theta$, which will be close to $\pi$ in any case. However, when $\left|J_{\text {imp }}\right| / J_{c}$ is close to the threshold of 1 , an effective reduction of $J_{\mathrm{c}}$ (which is expected for superlattices that favor ferromagnetic Ising order) leads to an increase of $\Delta \theta$. For example, in Fig. 5(a), the value of the canting angle would $\Delta \theta=1.02$ according to Eq. (3.15), but increases to $\Delta \theta=1.15$ after correcting it by Eq. (4.9).

Similar results are found for other superlattices. For instance, Figs. 5(c) and 5(d) show results for a denser superlattice, but with the same exchange couplings as in Figs. 5(a) and 5(b), respectively. In all panels of Fig. 5, the deviations from the local ferromagnetic order at nonimpurity bonds are small, justifying a posteriori the spin-wave approximation used to derive the effective Ising Hamiltonian (4.4).

To quantify the quality of the approximations incurred when trading the microscopic Hamiltonian (4.8) for the effective Ising Hamiltonian (4.4), we compare the quantity

$$
P:=\frac{1}{L_{x} L_{y}\left(L_{z}-1\right)} \sum_{\substack{\boldsymbol{r} \in \Lambda \\ 1 \leqslant r_{z} \leqslant L_{z}-1}} \sin \left(\phi_{\boldsymbol{r}+z}-\phi_{\boldsymbol{r}}\right),
$$

obtained from both Hamiltonians for several superlattices and various ratios $\left|J_{\text {imp }}\right| / J_{\perp}$ in Fig. 6(a). Here $L_{x}, L_{y}$, and $L_{z}$ in Eq. (4.11) are the linear dimensions of the lattice, while $P$ is an order parameter for the magnetic spiral phase. On the right-hand side, the sine of the relative angle between $\hat{S}_{r}$ and $\hat{S}_{r+z}$ is summed over all sites of the cubic host lattice $\Lambda$.

Figure 6(a) shows how the value of $|P|$, evaluated on the minimal-energy configuration, increases with increasing $\left|J_{\text {imp }}\right| / J_{\perp} \geqslant J_{\mathrm{c}} / J_{\perp}$ for three superlattices of impurity bonds in an isotropic cubic lattice $\left(J_{\|} / J_{\perp}=1\right)$, whereby all superlattices are chosen so that they induce a spiral state. At relatively large $\left|J_{\text {imp }}\right| / J_{\perp}$, the results for $|P|$ from the effective Ising Hamiltonian (4.4) (circles) are close to those obtained from the microscopic simulation of Hamiltonian (4.8) (squares), up to corrections of order $n_{\mathrm{imp}}$, as anticipated in the discussion around Eq. (3.28). However, as $\left|J_{\text {imp }}\right|$ approaches $J_{\mathrm{c}}$ from above, deviations become stronger, as we discussed after Eq. (4.9). In this regime the double-well potential defining the Ising degrees of freedom associated with the canting pattern becomes very shallow. Thus, even relatively weak contributions from neighboring impurities, $\Xi_{\tilde{r}}$, can stabilize and enhance the local canting $\Delta \theta$ and strengthen the spiral wave vector beyond the approximations we use to derive the effective model. For the same reason of mutual stabilization, we still find a finite spiral order $P \neq 0$ even when $J_{\text {imp }} / J_{\perp} \lesssim$ $J_{\mathrm{c}} / J_{\perp}=2$.

\section{Spiral phase in a realistic model for $\mathrm{YBaCuFeO}_{5}$}

It was argued in Ref. [19] that the magnetic degrees of freedom in the insulator $\mathrm{YBaCuFeO}_{5}$ (and analogously for 
related layered perovskites) realize a close cousin of the Hamiltonian (4.8), in that $J_{\perp}>0$ is to be replaced by two distinct values $J_{\perp}^{\prime}>0$ and $J_{\perp}^{\prime \prime}>0$ depending on the parity of the $z$ component of the coordinate $r$ of the cubic lattice. From the estimates for $J_{\|}>0, J_{\perp}^{\prime}>0, J_{\perp}^{\prime \prime}>0$, and $J_{\text {imp }}<0$ made in Ref. [19] we have borrowed the values

$$
\begin{aligned}
J_{\|} & =28.9 \mathrm{meV}, \\
J_{\text {imp }} & =-95.8 \mathrm{meV}, \\
J_{\perp} & \equiv\left(J_{\perp}^{\prime}+J_{\perp}^{\prime \prime}\right) / 2=4.1 \mathrm{meV} .
\end{aligned}
$$

Figure 6(b) compares the dependence of the magnitude $|P|$ of the spiral order parameter $P$ defined in Eq. (4.11) on $J_{\text {imp }}$ for the microscopic Hamiltonian (4.8) (squares) with that for the effective Ising Hamiltonian (4.4) (circles) for the case when the impurity bonds form a superlattice that stabilizes a longrange spiral order. Again, good agreement is found once the impurity bond strength is sufficiently stronger than the threshold, in which case the two possible canting patterns form robust local minima of the Hamiltonian. This is indeed the case in $\mathrm{YBaCuFeO}_{5}$, where $\left|J_{\mathrm{imp}}\right| / J_{\perp} \approx 23>J_{\mathrm{c}} / J_{\perp} \approx 9.4$. In Ref. [19] we have reported numerical finite-temperature results for such a model. Those showed the expected continuous phase transition from a collinear ferromagnetic phase to a low-temperature spiral state.

\section{Dependence of the ground state on the superlattice of impurity bonds}

We now illustrate how the choice made for the superlattice of impurity bonds affects the ground state. We use again the values (4.12) corresponding to ideal $\mathrm{YBaCuFeO}_{5}$ when defining the effective Ising Hamiltonian (4.4) and the microscopic Hamiltonian (4.8).

We consider two superlattices of impurity bonds. They are chosen such that $\Upsilon_{\boldsymbol{q}}$ has a global minimum at $\boldsymbol{q}_{\text {min }}=0$ for one and at $\boldsymbol{q}_{\text {min }}=\boldsymbol{C}^{\star} / 2$ for the other (cf. Fig. 7). Furthermore, both superlattices are chosen such that they share with $\mathrm{YBaCuFeO}_{5}$ the additional property that impurity bonds only occur between every other plane (namely, the planes hosting bipyramids).

The essential difference between the two superlattices lies in the relative position of nearest-neighbor impurity bonds. In the first lattice, the majority of nearest-neighbor impurity bonds is ferromagnetically coupled. This favors a ferromagnetic Ising phase in the effective Ising Hamiltonian (4.4), i.e., a spiral magnetic phase. In the second lattice, the majority of nearest-neighbor impurity bonds is antiferromagnetically coupled. This favors a layered antiferromagnetic Ising ground state of the effective Ising Hamiltonian (4.4) and thus a fanlike magnetic order with no net winding of the spins, whereby the orientation of the magnetization of the layers alternate between even and odd pairs of planes. We have verified using MC simulations of the microscopic Hamiltonian (4.8) that the long-range spiral order, which is present when the impurity bonds are arranged in certain Bravais superlattices, is robust to weak distortions of that superlattice, as expected on theoretical grounds.

\section{E. Limit of dilute impurities}

Next we analyze the limits of very dilute tetragonal, facecentered-cubic, and body-centered-cubic superlattices of impurity bonds. These are tractable analytically. We will show that ferromagnetic order prevails at the level of the Ising degrees of freedom associated with local cantings for dilute face-centered-cubic and body-centered-cubic superlattices of impurity bonds. This is to say that spiral order for the underlying $X Y$ spin degrees of freedom prevails for these diluted superlattices of impurity bonds. The results obtained here will also be useful for the study of random impurity bonds in Sec. V.

\section{Cubic superlattices}

We assume that the impurity bonds occupy a cubic sublattice $\mathcal{L}$ of the cubic host lattice $\Lambda$. As it turns out, this case supports antiferromagnetic order in the Ising model. Some results of this calculation will later help us to establish that the disordered case, in contrast, orders ferromagnetically.

If the cubic host lattice $\Lambda$ and the superlattice $\mathcal{L}$ are finite and not too large, it is possible to calculate the energy (3.38a) for all Ising spin configurations by exact evaluation of the Ising kernel $J_{\tilde{\boldsymbol{r}}-\tilde{\boldsymbol{r}}^{\prime}}^{(1)}$ defined in Eq. (3.38c). In the thermodynamic limit $|\Lambda| \rightarrow \infty$, with $n_{\text {imp }}$ held fixed, this approach is not possible anymore. Instead, we will restrict ourselves to a few long-range-ordered Ising configurations that are likely candidates for the ground state and compare their energies.

The ferromagnetic Ising configuration is described by

$$
\sigma_{\tilde{r}}^{F}:=1, \quad \frac{1}{|\Lambda|} \sum_{\tilde{r} \in \mathcal{L}} \sigma_{\tilde{r}}^{F}=n_{\mathrm{imp}}
$$

The most relevant competing states have ferromagnetic order in plane (as favored by the ferromagnetic interactions in the $x-y$ plane), but antiferromagnetic order along the $z$ axis. We consider the family of states defined by $[\tilde{\boldsymbol{r}}=(\tilde{x}, \tilde{y}, \tilde{z})]$

$$
\sigma_{\tilde{\boldsymbol{r}}}^{\mathrm{AF}(m)}:=(-1)^{\lfloor\tilde{z} / m \ell\rfloor}, \quad \frac{1}{|\Lambda|} \sum_{\tilde{\boldsymbol{r}} \in \mathcal{L}} \sigma_{\tilde{\boldsymbol{r}}}^{\mathrm{AF}(m)}=0,
$$

which describes a sequence of stacks of $m \geqslant 1$ layers, whose magnetization alternates. Here

$$
\ell \equiv n_{\text {imp }}^{-1 / 3}
$$

denotes the lattice spacing of the cubic superlattice and $\lfloor\tilde{z} / m \ell\rfloor$ returns the integer part of the fraction $\tilde{z} / m \ell$.

After subtraction of the three constants $E_{\mathrm{FM}}, E(\Delta \theta)|\mathcal{L}|$, and $-\gamma n_{\text {imp }} / 2 J_{\perp}$ on the right-hand side of Eq. (3.38a), the energy per impurity bond of the configurations $C \in\{F, \mathrm{AF}(m)\}$ is given by

$$
\varepsilon_{\mathcal{L}}^{C}=-\frac{\gamma}{2}\left(\sum_{\tilde{\boldsymbol{r}} \in \mathcal{L} \backslash\{\mathbf{0}\}} \Gamma_{\tilde{\boldsymbol{r}}}^{(0)} f^{C}(\tilde{z})+\frac{n_{\mathrm{imp}}}{J_{\perp}} \delta_{\mathrm{C}, \mathrm{F}}\right),
$$




\section{Spiral state}

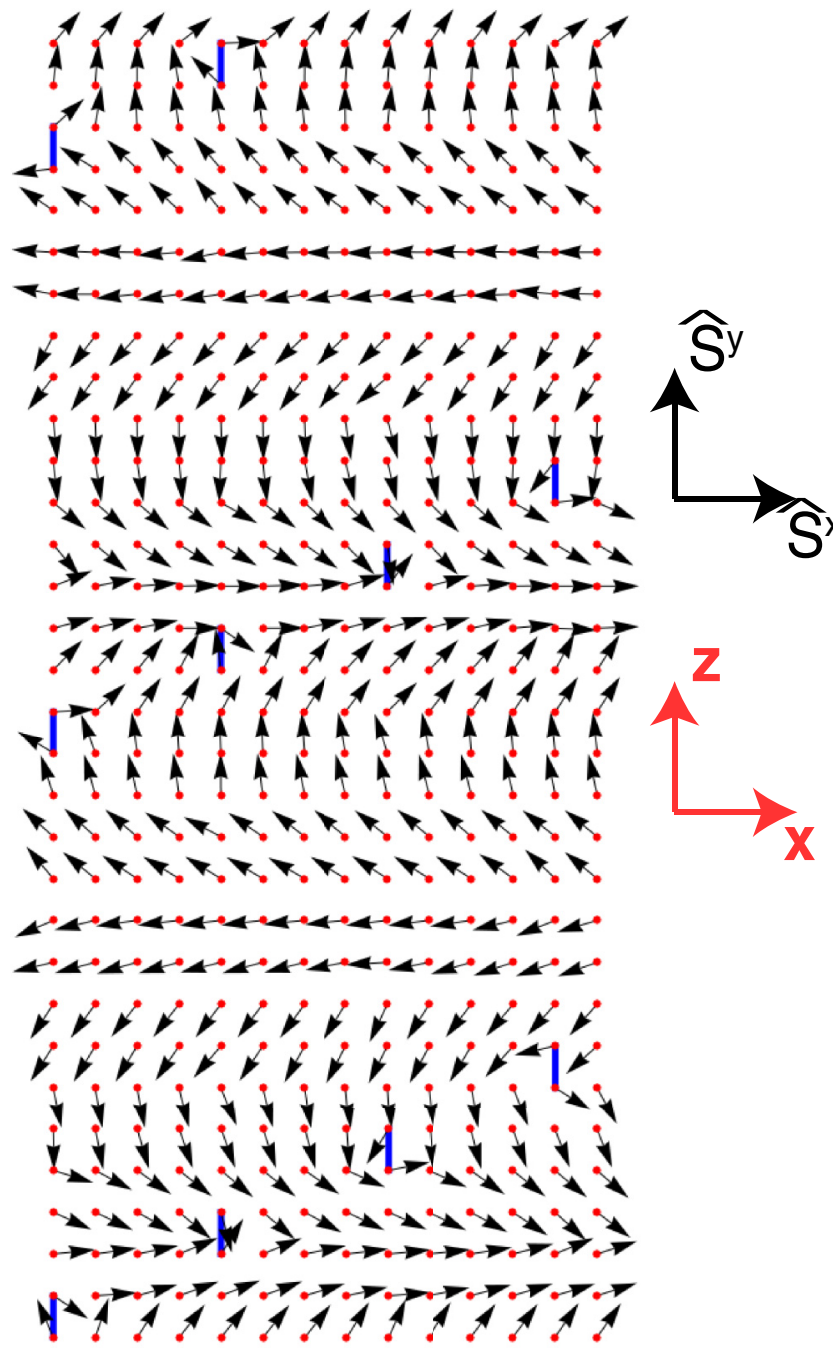

\section{Nonspiral (fanlike) state}

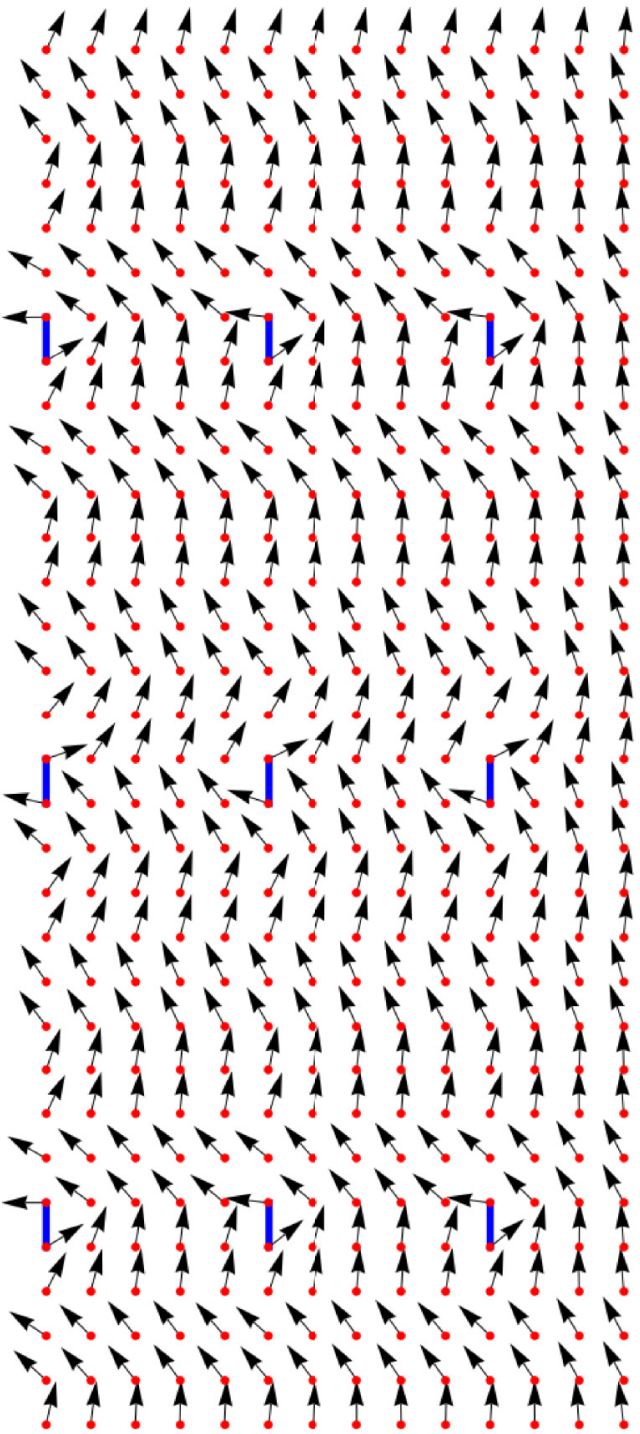

FIG. 7. Ground states obtained from the microscopic Hamiltonian (4.8). We choose the values of the exchange couplings motivated by those calculated for $\mathrm{YBaCuFeO}_{5}$ [cf. Eq. (4.12)] and with impurity bonds forming a regular superlattice. The ground state depends qualitatively on the superlattice. (a) A superlattice with basis vectors $\boldsymbol{A}=(4,3,0)^{\top}, \boldsymbol{B}=(0,4,2)^{\top}$, and $\boldsymbol{C}=(4,0,2)^{\top}\left(n_{\mathrm{imp}}=1 / 56\right)$ results in a magnetic spiral. (b) A superlattice with basis vectors $\boldsymbol{A}=(5,0,0)^{\top}, \boldsymbol{B}=(0,5,0)^{\top}$, and $\boldsymbol{C}=(0,1,2)^{\top}\left(n_{\text {imp }}=1 / 50\right)$ results in a nonspiral (fanlike) ground state. In this case, $\Upsilon_{\boldsymbol{q}}$ [Eq. (4.4c)] takes its minimum at $\boldsymbol{q}_{\min }=\boldsymbol{C}^{\star} / 2$, which yields a nonspiral ground state (with $Q=0$ ).

where the spin autocorrelation function

$$
f^{C}(\tilde{\boldsymbol{r}}) \equiv\left\langle\sigma_{\tilde{\boldsymbol{r}}^{\prime}} \sigma_{\tilde{\boldsymbol{r}}^{\prime}+\tilde{\boldsymbol{r}}}\right\rangle_{\tilde{\boldsymbol{r}}^{\prime}}=f^{C}(\tilde{z})
$$

only depends on the difference in the $\tilde{z}$ coordinate, owing to Eq. (4.13). Here $\langle\cdots\rangle_{\tilde{\boldsymbol{r}}^{\prime}}$ denotes the average over the sites $\tilde{\boldsymbol{r}}^{\prime}$ of the superlattice $\mathcal{L}$. For configurations $F$ and $\mathrm{AF}(1)$, it is given by

$$
f^{F}(\tilde{z})=1, \quad f^{\mathrm{AF}(1)}(\tilde{z})=(-1)^{\tilde{z} / \ell} .
$$

In the dilute limit $n_{\text {imp }} \rightarrow 0$, the typical distance between a pair of nearest-neighbor impurities is large. Hence, the typical pairwise interaction $\Gamma_{\tilde{r}}^{(0)}$ tends to the dipolar form (2.15) and can be safely used to evaluate $\varepsilon_{\mathcal{L}}^{\mathrm{AF}(m)}$ up to corrections which are subleading in the limit $n_{\text {imp }} \rightarrow 0$. The case of the ferromagnetic configuration is more subtle however. Indeed, a naive use of Eq. (2.15) would suggest that the first term on the right-hand side of Eq. (4.14a) vanishes due to the sum over symmetry related directions, while in fact it does not. This is due to corrections to the dipolar interaction (2.15) that scale as the inverse of the volume, but add up to a finite contribution when summed with equal signs over the whole superlattice. In the case of an isotropically shaped cubic sample with $L_{x}=L_{y}=L_{z}$ and isotropic interactions $J_{\|}=J_{\perp} \equiv J$, the computation can be done exactly, using the fact that upon averaging over all the permutations $k_{x} \rightarrow k_{y} \rightarrow$ $k_{z} \rightarrow k_{x}$ the kernel $\hat{\Gamma}_{\boldsymbol{k}}^{(0)}$ [Eq. (2.14b)] reduces to $1 / 3 J$. Using 
$f^{F}(z)=1$, this allows us to evaluate the lattice sum exactly for any impurity density as

$$
\begin{aligned}
\sum_{\tilde{\boldsymbol{r}} \in \mathcal{L} \backslash\{\mathbf{0}\}} \Gamma_{\tilde{\boldsymbol{r}}}^{(0)} f^{F}(\tilde{z}) & =\frac{1}{3 J|\Lambda|} \sum_{\tilde{\boldsymbol{r}} \in \mathcal{L} \backslash\{\mathbf{0}\}} \sum_{\boldsymbol{k} \in \mathrm{BZ}(\Lambda) \backslash\{\mathbf{0}\}} e^{i \boldsymbol{k} \cdot \tilde{\boldsymbol{r}}} \\
& =\frac{1}{3 J|\Lambda|} \sum_{\tilde{\boldsymbol{r}} \in \mathcal{L} \backslash\{\mathbf{0}\}}\left(|\Lambda| \delta_{\tilde{\boldsymbol{r}}, \mathbf{0}}-1\right) \\
& =-\frac{1}{3 J} \frac{|\mathcal{L}|-1}{|\Lambda|} \\
& =-\frac{n_{\text {imp }}}{3 J}+O\left(\frac{1}{|\Lambda|}\right) .
\end{aligned}
$$

This finite negative contribution disfavors the ferromagnet, in analogy to demagnetizing factors known from standard magnetic dipolar systems. Restricting ourselves to the isotropic case and inserting Eq. (4.15) into Eq. (4.14a), we obtain the energy per impurity

$$
\varepsilon_{\mathcal{L}}^{F}=-\frac{1}{3} \frac{\gamma n_{\mathrm{imp}}}{J_{\perp}} .
$$

More generally, it is useful to cast the energy (4.14a) for the ferromagnetic configuration (4.13a) of the Ising variables in a different form, namely,

$$
\begin{aligned}
\frac{\varepsilon_{\mathcal{L}}^{F}}{(\gamma / 2)} & =-\sum_{\tilde{\boldsymbol{r}} \in \mathcal{L}} \Gamma_{\tilde{\boldsymbol{r}}}^{(0)}+\Gamma_{\tilde{\boldsymbol{r}}=\mathbf{0}}^{(0)}-\frac{n_{\mathrm{imp}}}{J_{\perp}} \\
& =-\frac{1}{|\Lambda|} \sum_{\tilde{\boldsymbol{r}} \in \mathcal{L}} \sum_{\boldsymbol{k} \in \mathrm{BZ}(\Lambda)} e^{i \boldsymbol{k} \cdot \tilde{\boldsymbol{r}}} \hat{\Gamma}_{\boldsymbol{k}}^{(0)}+\Gamma_{\tilde{\boldsymbol{r}}=\mathbf{0}}^{(0)}-\frac{n_{\mathrm{imp}}}{J_{\perp}} \\
& =-\frac{|\mathcal{L}|}{|\Lambda|} \sum_{\substack{\boldsymbol{k} \in \mathrm{BZ}(\Lambda) \\
\boldsymbol{k} \in \mathcal{L}^{\star}}} \hat{\Gamma}_{\boldsymbol{k}}^{(0)}+\Gamma_{\tilde{\boldsymbol{r}}=\mathbf{0}}^{(0)}-\frac{n_{\mathrm{imp}}}{J_{\perp}}
\end{aligned}
$$

where the reciprocal lattice $\mathcal{L}^{\star}$ of $\mathcal{L}$ enters through the identity (4.3), $\hat{\Gamma}_{\boldsymbol{k} \neq \mathbf{0}}^{(0)}$ has been defined in Eq. $(2.14 \mathrm{~b})$, and we recall that $\hat{\Gamma}_{\boldsymbol{k}=\mathbf{0}}^{(0)}=0$. The sum over $\boldsymbol{k}$ thus contains $|\Lambda| /|\mathcal{L}|-1=$ $1 / n_{\mathrm{imp}}-1$ terms. Note that the self-interaction $\Gamma_{\tilde{r}=\mathbf{0}}^{(0)}$ [cf. Eq. (3.18)], which appears also in the single impurity energy, is subtracted on the right-hand side of Eq. (4.17).

We point out an important difference between the present effective dipolar problem and genuine magnetic dipoles. Genuine dipolar interactions are mediated by magnetic fields which extend everywhere in space, beyond the boundaries of the sample. Therefore, they only depend on the relative position of two spins, irrespective of where the spins are deep in the bulk or close to a surface of a finite sample. However, this is not so in our case where the dipolar interactions arise through the mediation of spin waves, which are confined to the sample. Accordingly, the interactions involving Ising spins at the periphery of the sample are not exactly the same as those for bulk Ising spins with the same relative position. More importantly, there are no magnetic stray fields beyond the sample. In real dipolar magnets those store a lot of magnetic energy, which is avoided in the ground state by domain formation. The unavoidable presence of domains complicates the computation of the energy density. In particular, the evaluation for a homogeneously magnetized sample yields a shape-dependent result, a fact that is reflected in the ambiguity of the value of the Fourier transform of the dipolar interactions (2.14b) in the limit $\boldsymbol{k} \rightarrow 0$. In the present case, however, such problems do not arise, since the spin-wave-mediated interaction is such that $\hat{\Gamma}_{\boldsymbol{k}=\mathbf{0}}^{(0)}=0$. This eliminates the potential ambiguity and therefore eliminates the shape dependence. We also do not expect the effective dipolar interactions to induce domains, in contrast to genuine ferromagnets.

Performing a calculation analogous to the one above yields, for the antiferromagnet $\mathrm{AF}(1)$,

$$
\begin{aligned}
\frac{\varepsilon_{\mathcal{L}}^{\mathrm{AF}(1)}}{\gamma / 2} & =-\sum_{\tilde{\boldsymbol{r}} \in \mathcal{L}} \Gamma_{\tilde{\boldsymbol{r}}}^{(0)}(-1)^{\lfloor\tilde{z} / \ell\rfloor}+\Gamma_{\tilde{\boldsymbol{r}}=\mathbf{0}}^{(0)} \\
& =-\frac{|\mathcal{L}|}{|\Lambda|} \sum_{\substack{\boldsymbol{k} \in \mathrm{BZ}(\Lambda) \\
k+(0,0, \pi / \ell)^{\top} \in \mathcal{L}^{\star}}} \hat{\Gamma}_{\boldsymbol{k}}^{(0)}+\Gamma_{\tilde{\boldsymbol{r}}=\mathbf{0}}^{(0)} .
\end{aligned}
$$

Even though the quantitative mapping from the $X Y$ Hamiltonian (2.1) to the effective Ising Hamiltonian (3.38) only holds for low densities of impurity bonds, it is useful to study the effective Ising Hamiltonian (3.38) in its own right, i.e., without requiring the impurity bonds to be dilute.

A maximally dense superlattice is defined by

$$
\ell=1, \quad \mathcal{L}=\Lambda, \quad n_{\text {imp }}=\frac{|\mathcal{L}|}{|\Lambda|}=1 .
$$

For such a lattice, one finds the ferromagnetic $F$ and antiferromagnetic $\mathrm{AF}(1)$ states to be degenerate,

$$
\frac{\varepsilon_{\mathcal{L}}^{F}-\varepsilon_{\mathcal{L}}^{\mathrm{AF}(1)}}{(\gamma / 2) n_{\mathrm{imp}}}=\hat{\Gamma}_{\boldsymbol{k}=(0,0, \pi)^{\top}}^{(0)}-\hat{\Gamma}_{\boldsymbol{k}=\mathbf{0}}^{(0)}-\frac{1}{J_{\perp}}=0,
$$

since [cf. Eqs. (2.14b) and (2.14c)]

$$
\hat{\Gamma}_{\left(0,0, k_{z}\right)^{\top}}^{(0)}=\frac{1-\delta_{k_{z}, 0}}{J_{\perp}} .
$$

The identity (4.21) obeyed by the kernel (2.14b) can be used together with the expression (4.4a) and the fact that only $\boldsymbol{q}$ of the form $\left(0,0, k_{z}\right)^{\top}$ enter it, to show that for a maximally dense superlattice all antiferromagnetic states $\mathrm{AF}(m)$ are degenerate with the ferromagnet. More generally, it is shown in the Appendix that the ferromagnet is degenerate with any Ising configuration in which the spins in every given plane at fixed $z$ coordinate are ferromagnetically aligned, irrespective of the relative orientation of the magnetization of different planes.

This degeneracy is however lifted at finite dilution, where the way in which the dilution is realized is crucial. For example, diluting the impurity density $n_{\text {imp }}$ by maintaining a cubic superlattice but increasing its integer lattice spacing $\ell$ disfavors the ferromagnetic state. This is illustrated in Fig. 8, where we plot the energies per impurity as a function of superlattice spacing $\ell$. For small $\ell$, the energy difference is obtained from the representations (4.17) and (4.18). In the dilute limit, $n_{\text {imp }}=\ell^{-3} \rightarrow 0$, the reciprocal lattice $\mathcal{L}^{\star}$ only contains small wave vectors, and we may replace $1-\cos k_{i}$ 


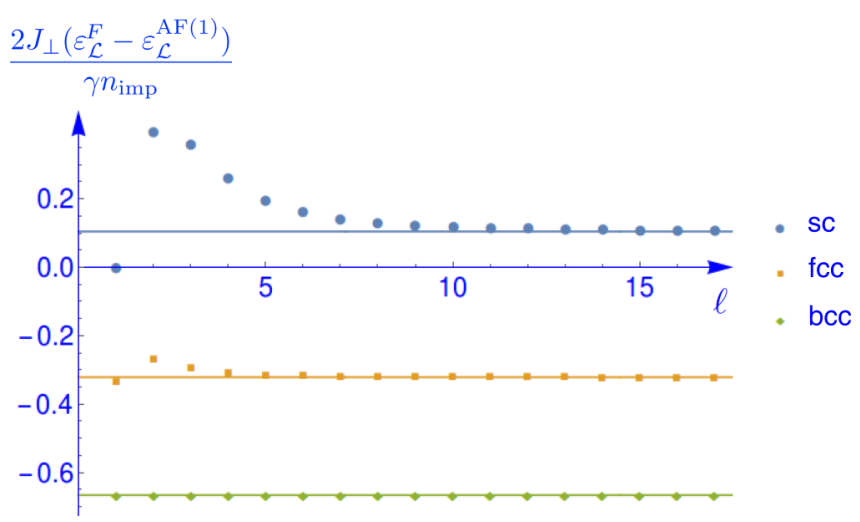

FIG. 8. Dependence of the energy difference between the Ising ferromagnetic $F$ and antiferromagnetic $\mathrm{AF}(1)$ states on the linear size $\ell$ of the unit cell of the superlattice for different classes of superlattices. Blue circles represent a simple cubic (sc) superlattice with the basis vectors $(\ell, 0,0),(0, \ell, 0)$, and $(0,0, \ell)$. Yellow squares represent a face-centered-cubic (fcc) superlattice with lattice vectors $(\ell, \ell, 0),(\ell, 0, \ell)$, and $(0, \ell, \ell)$. Green diamonds represent bodycentered-cubic (bcc) superlattices with the lattice vectors $(\ell, \ell, \ell)$, $(\ell,-\ell, \ell)$, and $(\ell, \ell,-\ell)$. The correspondingly colored horizontal solid lines represent the dilute limit $\ell \rightarrow \infty$ of these energy differences for each superlattice.

(where $k_{i}=2 \pi n_{i} / \ell$ ) in the kernel $(2.14 \mathrm{~b})$ by $\left(2 \pi n_{i}\right)^{2} / 2 \ell^{2}$ with $n_{i} \in \mathbb{Z}$ for $i=x, y, z$, i.e.,

$$
\frac{\varepsilon_{\mathcal{L}}^{F}-\varepsilon_{\mathcal{L}}^{\mathrm{AF}(1)}}{(\gamma / 2) n_{\mathrm{imp}}} \rightarrow \frac{\delta(\alpha)}{J_{\perp}},
$$

where the exchange anisotropy parameter $\alpha$ was defined in Eq. (3.21) and

$$
\begin{aligned}
\delta(\alpha):= & -1-\sum_{\boldsymbol{n} \in \mathbb{Z}^{3} \backslash\{\mathbf{0}\}} \frac{n_{z}^{2}}{\alpha\left(n_{x}^{2}+n_{y}^{2}\right)+n_{z}^{2}} \\
& +\sum_{\boldsymbol{n} \in \mathbb{Z}^{3}} \frac{\left(n_{z}-1 / 2\right)^{2}}{\alpha\left(n_{x}^{2}+n_{y}^{2}\right)+\left(n_{z}-1 / 2\right)^{2}} .
\end{aligned}
$$

The sum over $n_{z}$ can be carried out explicitly,

$$
\delta(\alpha)=\sum_{\boldsymbol{n} \in \mathbb{Z}^{2} \backslash\{\boldsymbol{0}\}} \frac{2 \pi \sqrt{\alpha\left(n_{x}^{2}+n_{y}^{2}\right)}}{\sinh \left[2 \pi \sqrt{\alpha\left(n_{x}^{2}+n_{y}^{2}\right)}\right]} .
$$

Hence, $\delta(\alpha)$ is always positive. For the isotropic limit $\alpha=1$, one finds $\delta(1) \approx 0.1042$.

Alternatively, one can calculate the antiferromagnetic energy directly in real space using the dipolar form (2.15). This can be used to calculate the energies of other antiferromagnetic states $\operatorname{AF}(m)$, which all scale as

$$
\frac{\varepsilon_{\mathcal{L}}^{\mathrm{AF}(m)}}{(\gamma / 2) n_{\mathrm{imp}}}=-\frac{c_{m}}{J_{\perp}} .
$$

From the results (4.16) and (4.22a) it follows that $c_{1}=\delta(1)+$ $2 / 3$, while one finds the higher $c_{m}$ 's to decrease monotonically with increasing $m$. From this we conclude that a dilute cubic superlattice orders antiferromagnetically with layer magnetizations that alternate in sign $(m=1)$.

\section{Dilute, face-centered, and body-centered tetragonal superlattices}

One readily generalizes the above calculation to tetragonal superlattices $\mathcal{L}$ with unit vectors $(A \ell, 0,0)^{\mathrm{T}},(0, A \ell, 0)^{\mathrm{T}}$, and $(0,0, C \ell)^{\top}$, where $A$ and $C$ are fixed integers while the integervalued dilution parameter $\ell$ will be taken to infinity. This case is obtained from that of a cubic lattice by substituting

$$
n_{\mathrm{imp}} \rightarrow \frac{1}{A^{2} C \ell^{3}}, \quad \alpha \rightarrow \frac{J_{\|}}{J_{\perp}} \frac{C^{2}}{A^{2}}
$$

in Eqs. (4.22a) and (4.22b). Independently of the ratio $C / A$ of the tetragonal superlattice, the Ising antiferromagnetic state $\mathrm{AF}(1)$ is favored over the Ising ferromagnetic state $F$.

However, similarly as in lattice problems of physical electric or magnetic dipoles [29] where the interactions have reversed global sign, a different ground state is found in dilute body-centered or face-centered tetragonal lattices. The difference arises because closest neighbors in these lattices have a stronger tendency to have ferromagnetic interactions than in simple tetragonal lattices. For the face-centered tetragonal lattice, the basis vectors are $(A, A, 0),(A, 0, C)$, and $(0, A, C)$. The corresponding dual basis vectors in reciprocal space are $\boldsymbol{e}_{1}=\pi(1 / A, 1 / A,-1 / C), \boldsymbol{e}_{2}=\pi(1 / A,-1 / A, 1 / C)$, and $\boldsymbol{e}_{3}=$ $\pi(-1 / A, 1 / A, 1 / C)$. Their linear combinations with integer coefficients span the reciprocal lattice $\mathcal{L}^{\star}$. It is convenient to represent a generic reciprocal lattice vector $\boldsymbol{G} \in \mathcal{L}^{\star}$ as $\boldsymbol{G}=$ $n_{1} \boldsymbol{e}_{1}+n_{2} \boldsymbol{e}_{2}+n_{3}\left(\boldsymbol{e}_{2}+\boldsymbol{e}_{3}\right)$. With this choice, the asymptotic energy difference between the ferromagnetic and the antiferromagnetic states in the infinite dilution limit $n_{\text {imp }} \rightarrow 0$ can be written as

$$
\frac{\varepsilon_{\mathcal{L}}^{F}-\varepsilon_{\mathcal{L}}^{\mathrm{AF}(1)}}{(\gamma / 2) n_{\mathrm{imp}}}=-\frac{1}{J_{\perp}}-\sum_{\boldsymbol{n} \in \mathbb{Z}^{3} \backslash\{\boldsymbol{0}\}} \frac{g_{\boldsymbol{n}}}{J_{\perp}}+\sum_{\boldsymbol{n} \in \mathbb{Z}^{3}} \frac{g_{\left(n_{1}, n_{2}, n_{3}+1 / 2\right)}}{J_{\perp}},
$$

where

$$
g_{\boldsymbol{n}}:=\frac{\left(n_{1}-n_{2}-2 n_{3}\right)^{2}}{\alpha\left[\left(n_{1}+n_{2}\right)^{2}+\left(n_{1}-n_{2}\right)^{2}\right]+\left(n_{1}-n_{2}-2 n_{3}\right)^{2}},
$$

where for vanishing wave vector we have to set $g_{0}=0$. Carrying out the sum over $n_{3}$, one finds

$$
\frac{\varepsilon_{\mathcal{L}}^{F}-\varepsilon_{\mathcal{L}}^{\mathrm{AF}(1)}}{(\gamma / 2) n_{\mathrm{imp}}}=\sum_{\boldsymbol{n} \in \mathbb{Z}^{2} \backslash\{\mathbf{0}\}} \frac{(-1)^{n_{1}-n_{2}}}{J_{\perp}} \frac{\pi \sqrt{2 \alpha\left(n_{1}^{2}+n_{2}^{2}\right)}}{\sinh \left(\pi \sqrt{2 \alpha\left(n_{1}^{2}+n_{2}^{2}\right)}\right)} .
$$

In the isotropic case $\alpha=1$, the energy difference is negative $J\left(\varepsilon_{\mathcal{L}}^{F}-\varepsilon_{\mathcal{L}}^{\mathrm{AF}(1)}\right) /(\gamma / 2) n_{\mathrm{imp}}=-0.3218$, so the ferromagnetic order prevails. For body-centered tetragonal lattices one finds the same expression, but with the replacement $2 \alpha \rightarrow \alpha$. The energy difference turns out to be always negative for any value of $\alpha$, as seen in Fig. 9. Thus, in both these types of superlattices the ferromagnetic state is favored over the layered antiferromagnetic state, whatever the tetragonal aspect ratio.

\section{RANDOM IMPURITIES: DILUTE LIMIT}

In this section we study randomly distributed impurities that occupy a fraction $n_{\mathrm{imp}}$ of the sites of the cubic host 

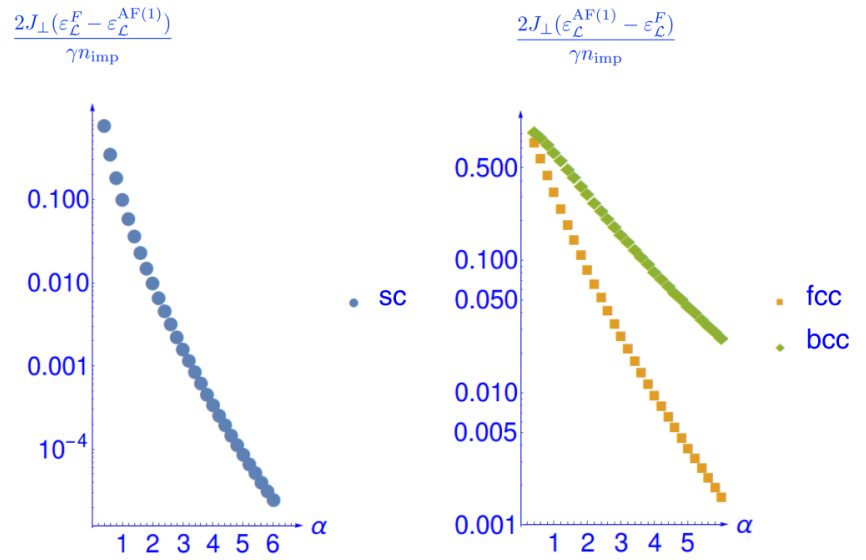

FIG. 9. Shown on the right is the dependence of the energy difference between the Ising ferromagnetic state $F$ and antiferromagnetic state $\mathrm{AF}(1)$ as a function of $\alpha \equiv J_{\|} / J_{\perp}$ [recall Eq. (3.21)] for a simple cubic superlattice in the large dilution limit. On the left is the energy difference between the Ising antiferromagnetic state $\mathrm{AF}(1)$ and ferromagnetic state $F$ for face-centered superlattices (yellow squares) and a body-centered superlattice (green diamonds). Independently of the value of $\alpha$, the antiferromagnetic state has lower energy for simple cubic superlattices while the ferromagnetic state has lower energy for face- and body-centered superlattices.

lattice $\Lambda$. We assume again that the relevant contenders for the ground state are given by Eqs. (4.13a) and (4.13b). In Eq. (4.13b) we set $\ell=1$, since only the lattice constant of the cubic host lattice $\Lambda$ is relevant. These configurations are expected to come reasonably close to the true ground state and the relevant competing metastable configurations. However, they will differ in the orientation of a few spins from the simple configurations (4.13a) and (4.13b). The relative fraction of these spins becomes increasingly small as $n_{\text {imp }} \rightarrow 0$, as discussed below.

If the impurities are distributed randomly according to a Poisson process, the average energy per impurity bond of the trial configurations $C=F, \mathrm{AF}(m)$ is given by

$$
\varepsilon_{\mathrm{dis}}^{C}=-\frac{\gamma}{2} n_{\mathrm{imp}}\left(\sum_{\boldsymbol{r} \in \Lambda} \Gamma_{r}^{(0)} f^{C}(z)+\frac{1}{J_{\perp}} \delta_{C, F}\right),
$$

since any site $\boldsymbol{r}$ of the cubic host lattice $\Lambda$ is the lower end of an impurity bond with probability $n_{\text {imp }}$, independently of the location of other impurities. From this observation, one might at first conclude that the antiferromagnetic state should dominate again. However, the above consideration does not treat correctly impurities located at short distances from each other. On the one hand, rare pairs of impurities that are located much closer to each other than the average separation $n_{\text {imp }}^{-1 / 3}$ do not follow the pattern (4.13a) and (4.13b), but simply optimize their mutual interaction energy, irrespective of the global ordering pattern. Since such pairs nevertheless contribute a finite fraction to the total energy estimated above, they must be corrected for, which will turn out to favor the ferromagnetic ordering. This conclusion will become clear below, as a corollary to the discussion of another short-distance effect, which we will consider first.

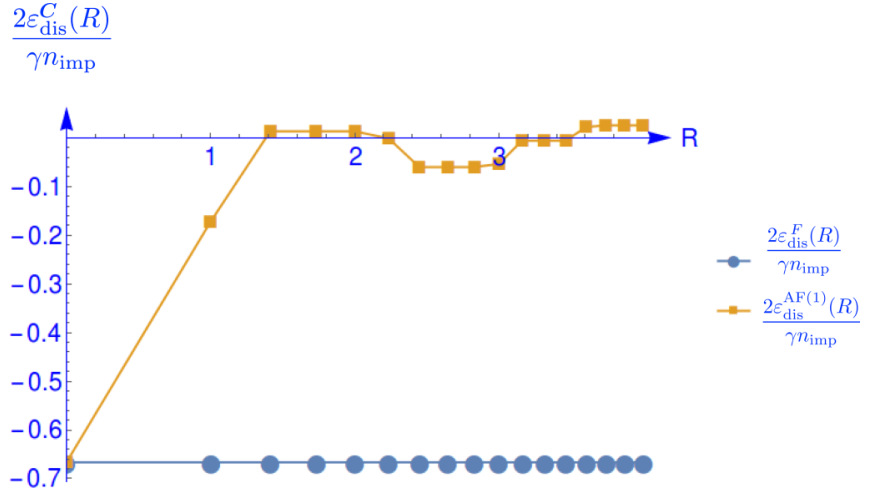

FIG. 10. Dependence on $R$ of $\varepsilon_{\text {dis }}^{C}(R) / \gamma n_{\text {imp }}$ defined in Eq. (5.2) for isotropic couplings $J_{\perp}=J_{\|} \equiv J$ for the ferromagnetic $(C=F$, blue dots) and the layered antiferromagnetic state $[C=\mathrm{AF}(1)$, yellow squares]. Energies are given in units of $J$.

Impurity distributions in real materials are usually not simply governed by a Poisson process, but rather one should expect them to exhibit some short-range correlations. For example, in the case of $\mathrm{YBaCuFeO}_{5}$ and related layered perovskites the impurity bonds arise due to chemical disorder which occasionally replaces the usual $\mathrm{Cu}-\mathrm{Fe}$ pairs on bonds along its crystallographic $c$ axis by impurity configurations consisting in $\mathrm{Fe}-\mathrm{Fe}$ or $\mathrm{Cu}-\mathrm{Cu}$ pairs. $\mathrm{Fe}-\mathrm{Fe}$ pairs differ from $\mathrm{Fe}-$ $\mathrm{Cu}$ pairs by the sign and magnitude of the resulting magnetic exchange constant. Moreover, both $\mathrm{Fe}-\mathrm{Fe}$ and $\mathrm{Cu}-\mathrm{Cu}$ pairs differ from $\mathrm{Fe}-\mathrm{Cu}$ pairs in their local charge density. The resulting Coulomb repulsion between such impurity configurations thus suppresses the occurrence of pairs of impurities at short distances. In a crude manner, we can mimic this effect by a hard constraint on the minimal distance between impurities, excluding distance vectors with $|\boldsymbol{r}| \leqslant R$. With such a constraint the average energy per impurity (5.1) is modified to

$$
\varepsilon_{\mathrm{dis}}^{C}(R)=-\frac{\gamma}{2} n_{\mathrm{imp}}\left(\sum_{\substack{r \in \Lambda \\|r|>R}} \Gamma_{r}^{(0)} f^{C}(z)+\frac{1}{J_{\perp}} \delta_{C, F}\right) .
$$

Note that for $R=0$ these energies are simply $n_{\text {imp }}$ multiplying the energy per impurity $\varepsilon_{\mathcal{L}=\Lambda}^{C}(R)$ of a maximally dense system of impurities [recall Eq. (4.14a)]. As we have shown in the preceding section, those energies are all degenerate. Since the sum over $\boldsymbol{r}$ in Eq. (5.2) is dominated by small $|\boldsymbol{r}|$, even a small $R$ of the order of one lattice constant will have a decisive effect and lifts this degeneracy. In Fig. 10 we plot as a function of $R$ the average energies $2 \varepsilon_{\text {dis }}^{F}(R) / \gamma n_{\text {imp }}$ and $2 \varepsilon_{\mathrm{dis}}^{\mathrm{AF}(m=1)}(R) / \gamma n_{\text {imp }}$ of the two most relevant competing states. Already, for the smallest effective exclusion radius of $R \geqslant R_{\mathrm{c}}=1$ (in units of the host cubic lattice spacing), we find that the ferromagnetic state (and thus $X Y$ spiral order) wins over the antiferromagnetic state, i.e., $X Y$ fan order. This numerical result can be understood by recalling that $\varepsilon^{F}$ and $\varepsilon^{\mathrm{AF}(1)}$ are degenerate for $R=0$. Upon barring impurities on nearest-neighbor sites on the host cubic lattice, the two states receive a relative energy shift $4 n_{\text {imp }} \Gamma_{r=z}=4 n_{\text {imp }}\left(\frac{A}{J}\right)$, which stabilizes the ferromagnetic state $(A \approx 0.123)$. Larger 
exclusion radii tend to reinforce this trend, as shown in Fig. 10. In the limit of large $R$, the energy per impurity bond of the ferromagnetic state is more favorable than that of the antiferromagnetic one by $\gamma n_{\text {imp }} / 3 J$ in the case of isotropic couplings. This can be understood as follows. For isotropic couplings, the ferromagnetic energy per bond, $\varepsilon^{F}=-\gamma n_{\text {imp }} / 3 J$, remains unchanged upon excluding the interactions with a set of sites that is invariant under the cubic symmetry group, as seen in Fig. 10. In contrast, in an antiferromagnetic state, the interactions with the neighbors in thin spherical shells of approximately fixed radius $r>R$ come with alternating signs. Those tend to cancel more effectively the larger $R$ is, such that $\varepsilon^{\mathrm{AF}(1)} / \gamma n_{\text {imp }} \rightarrow 0$ as $R \rightarrow \infty$.

Even without any repulsive short-range correlations between impurity locations, one expects Ising ferromagnetism to prevail at sufficiently low impurity densities. This is because rare impurities with a neighboring impurity much closer than $n_{\text {imp }}^{-1 / 3}$ should effectively be taken out of the calculation for the average energy. Indeed, if the close pair is antiferromagnetically coupled, it will antialign, have no net moment, and thus essentially decouples from the global ordering pattern. If instead the pair is ferromagnetically coupled, it forms a bigger spin that can then be incorporated in the consideration like any other typical spin. The net effect of treating such close pairs in this way is in essence a matter of considering only original or effective spins with pairwise separations of the order of $R_{\text {eff }} \gtrsim$ $c n_{\text {imp }}^{-1 / 3}$ with some constant $c$ of order 1 . The competition for the global ordering pattern then becomes essentially identical to the one of the constrained superlattice above, with $R_{\text {eff }}$ now taking the role of the exclusion radius in Eq. (5.2). From these considerations we predict that for sufficiently dilute concentrations $n_{\text {imp }} \lesssim(c / R)_{\mathrm{c}}^{3}$ the Ising ferromagnetic order prevails.

\section{FINITE-TEMPERATURE TRANSITION TO THE SPIRAL PHASE}

In Ref. [19] it was established numerically for $O(3)$ symmetric spin models described by Eq. (4.8) that a continuous phase transition to a spiral phase occurs when the impurities were randomly distributed and their density was not too high. In this section we aim at an analytical understanding of the phase transition as a function of the exchange couplings. This can be achieved in the limit of low impurity density.

The effective Ising model (3.38) undergoes ordering at a critical temperature $T_{\text {Ising }} \propto n_{\text {imp. }}{ }^{1}$ As long as $T_{\text {Ising }}$ lies in the

\footnotetext{
${ }^{1}$ One might worry that a critical temperature $T_{\text {Ising }}$ for the effective Ising model (3.38) is not well defined in the thermodynamic limit in view of the long-range nature of the kernel (3.38c). In particular, $T_{\text {Ising }}$ might depend on the aspect ratio of the lattice $\Lambda$ as $|\Lambda| \rightarrow \infty$. We argue that this is not the case as follows. Since the Hamiltonian (2.1) has only short-range magnetic interactions, any ordering temperature that it supports is well defined (independently of how the limit $|\Lambda| \rightarrow$ $\infty$ is taken) and of order unity, as guaranteed by Griffith's theorem [31]. We first rescale the coordinate axes and then take any reference shape for which a single ferromagnetic domain is expected, i.e., a prolate rather than a needlelike sample, so that we can safely assume a single global spiral to emerge. We then integrate out the spin
}

range of low temperatures (3.16), the Ising approximation is well justified. This is certainly the case for $n_{\text {imp }} \ll 1$. Since the reduction to the Ising model neglects some fluctuations, we expect $T_{\text {Ising }}$ to be an upper bound to the actual spiral transition temperature $T_{\mathrm{spi}}$. However, the bound should become increasingly tight as the impurity concentration decreases towards $n_{\text {imp }} \rightarrow 0$.

\section{A. Mean-field theory}

We first estimate $T_{\text {Ising }}$ using mean-field theory, which should work well as three-dimensional space is the upper critical dimension for the Ising model with dipolar interactions [30,32-35]. However, we will focus on the case of randomly distributed impurities, where the mean field actually depends on the site that is considered. This will require a number of additional approximations. In the next section, we will follow an alternative and complementary approach that instead makes use of the dipolar nature of the interactions and exploits their covariance under spatial rescalings. This allows us to predict how $T_{\text {Ising }}$ depends on the couplings, without resorting to a mean-field approximation.

To implement a mean-field treatment, we replace the Ising Hamiltonian (3.38) by the mean-field Hamiltonian

$$
H_{\mathcal{L}}^{\mathrm{MF}}:=-\sum_{\tilde{\boldsymbol{r}} \in \mathcal{L}} B_{\tilde{\boldsymbol{r}}}^{\mathrm{MF}} \sigma_{\tilde{\boldsymbol{r}}}
$$

where the effects on $\sigma_{\tilde{r}}$ from all the Ising spins $\sigma_{\tilde{r}^{\prime}}$ is approximately captured by the mean magnetic field

$$
B_{\tilde{r}}^{\mathrm{MF}}:=\sum_{\tilde{r}^{\prime} \in \mathcal{L} \backslash\langle\tilde{r}\}} J_{\tilde{r}-\tilde{r}^{\prime}}^{(\mathrm{I})}\left\langle\sigma_{\tilde{r}^{\prime}}\right\rangle_{\mathrm{MF}} .
$$

The mean-field magnetic moments $\left\langle\sigma_{\tilde{r}}\right\rangle_{\mathrm{MF}}$ are subject to the nonlinear constraint $\left(k_{\mathrm{B}}=1\right)$

$$
\left\langle\sigma_{\tilde{\boldsymbol{r}}}\right\rangle_{\mathrm{MF}}=\tanh \left(\frac{1}{T} \sum_{\tilde{\boldsymbol{r}}^{\prime} \in \mathcal{L} \backslash\{\tilde{\boldsymbol{r}}\}} J_{\tilde{\boldsymbol{r}}-\tilde{\boldsymbol{r}}^{\prime}}^{(\mathrm{I})}\left\langle\sigma_{\tilde{\boldsymbol{r}}^{\prime}}\right\rangle_{\mathrm{MF}}\right) .
$$

The mean-field transition temperature is obtained in two steps. First, we linearize the constraint (6.1c), assuming a small order parameter

$$
\left\langle\sigma_{\tilde{r}}\right\rangle_{\mathrm{MF}}=\frac{1}{T} \sum_{\tilde{r}^{\prime} \in \mathcal{L} \backslash\{\tilde{r}\}} J_{\tilde{r}-\tilde{r}^{\prime}}^{(\mathrm{I})}\left\langle\sigma_{\tilde{r}^{\prime}}\right\rangle_{\mathrm{MF}} .
$$

If translational symmetry were to hold and $\left\langle\sigma_{\tilde{r}}\right\rangle_{\mathrm{MF}}$ were independent of $\tilde{\boldsymbol{r}}$, the critical temperature

$$
T_{\mathrm{Ising}}^{\mathrm{MF}}=\sum_{\tilde{r} \in \mathcal{L} \backslash\{0\}} J_{\tilde{r}}^{(\mathrm{I})}
$$

would follow. However, translational symmetry breaks down when the impurity bonds are distributed randomly, in which

waves. In this way, we eventually end up with the global energy scale $\kappa n_{\text {imp }}$ multiplying a dimensionless Hamiltonian with unit density of impurity sites, as is done to obtain the Hamiltonians (6.11) and (6.14), the critical temperature of which serves as a reference for all antidipolar systems. 
case we estimate the critical temperature by the disorder average

$$
T_{\text {Ising }}^{\mathrm{MF}, \text { av }}:=\frac{1}{|\mathcal{L}|} \sum_{\tilde{\boldsymbol{r}} \in \mathcal{L}} \sum_{\tilde{\boldsymbol{r}}^{\prime} \in \mathcal{L} \backslash\{\boldsymbol{r}\}} J_{\tilde{\boldsymbol{r}}-\tilde{\boldsymbol{r}}^{\prime}}^{(\mathrm{I})}
$$

Substituting the definition of $J_{\tilde{r}-\tilde{r}^{\prime}}^{(\mathrm{I})}$ in Eq. (6.4) yields

$$
\begin{aligned}
T_{\text {Ising }}^{\mathrm{MF}, \mathrm{av}} & =\frac{\gamma}{|\mathcal{L}|} \sum_{\tilde{\boldsymbol{r}} \in \mathcal{L}} \sum_{\tilde{\boldsymbol{r}}^{\prime} \in \mathcal{L} \backslash\{\tilde{\boldsymbol{r}}\}}\left(\Gamma_{\tilde{\boldsymbol{r}}-\tilde{\boldsymbol{r}}^{\prime}}^{(0)}+\frac{1}{J_{\perp}|\Lambda|}\right), \\
& =\gamma n_{\mathrm{imp}}\left[\sum_{\boldsymbol{r} \in \Lambda \backslash\{\boldsymbol{0}\}} \Gamma_{\boldsymbol{r}}^{(0)}+\frac{1}{J_{\perp}}+O\left(\frac{1}{|\Lambda|}\right)\right], \\
& =\gamma n_{\mathrm{imp}}\left[-\Gamma_{\boldsymbol{r}=\mathbf{0}}^{(0)}+\frac{1}{J_{\perp}}+O\left(\frac{1}{|\Lambda|}\right)\right] \\
& =n_{\mathrm{imp}}(\Delta \theta)^{2} \frac{J_{c}}{J_{\perp}}\left(J_{c}+J_{\perp}\right)+O\left(\frac{1}{|\Lambda|}\right) .
\end{aligned}
$$

To reach the second equality, we have used the fact that in a random, uncorrelated set of points $\mathcal{L}$ the distance vectors $\tilde{\boldsymbol{r}}-\tilde{\boldsymbol{r}}^{\prime}$ appear with the same relative frequency as in the translationally invariant host lattice $\Lambda$. More precisely, we used

$$
\frac{1}{|\mathcal{L}|} \sum_{\tilde{\boldsymbol{r}} \in \mathcal{L}} \sum_{\tilde{\boldsymbol{r}}^{\prime} \in \mathcal{L} \backslash\{\tilde{\boldsymbol{r}}\}}(\cdots)=\frac{n_{\mathrm{imp}}}{|\Lambda|} \sum_{\boldsymbol{r} \in \Lambda} \sum_{\boldsymbol{r}^{\prime} \in \Lambda \backslash\{\boldsymbol{r}\}}(\cdots) .
$$

To reach the third equality, we have used that

$$
\sum_{r \in \Lambda} \Gamma_{r}^{(0)}=\hat{\Gamma}_{k=0}^{(0)}=0 .
$$

The fourth equality follows from the relations

$$
\gamma:=(\Delta \theta)^{2}\left(J_{\mathrm{c}}+J_{\perp}\right)^{2}, \quad \frac{1}{J_{\perp}}-\Gamma_{\boldsymbol{r}=\mathbf{0}}^{(0)}=\frac{J_{\mathrm{c}} / J_{\perp}}{J_{\mathrm{c}}+J_{\perp}}
$$

[see Eq. (3.18) for $\left.\Gamma_{r=0}^{(0)}\right]$.

Next we compare the transition temperature $T_{\mathrm{Ising}}^{\mathrm{MF} \text {,av }}$ with the absolute value of the spiral twist rate at zero temperature

$$
|Q|=\Delta \theta \frac{J_{\mathrm{c}}+J_{\perp}}{J_{\perp}} \frac{1}{|\Lambda|} \sum_{\tilde{\boldsymbol{r}} \in \mathcal{L}} \sigma_{\tilde{\boldsymbol{r}}} \leqslant \Delta \theta \frac{J_{\mathrm{c}}+J_{\perp}}{J_{\perp}} n_{\mathrm{imp}},
$$

as follows from Eq. (3.35). Equality holds when the canting degrees of freedom $\sigma_{\tilde{r}}$ order ferromagnetically. In that case we find that both the transition temperature and the twist rate of the spiral are proportional to the impurity concentration $n_{\text {imp }}$, with a ratio

$$
\frac{T_{\mathrm{Ising}}^{\mathrm{MF}, \mathrm{av}}}{|Q|}=\Delta \theta J_{\mathrm{c}}
$$

Note that this ratio is independent of $n_{\text {imp }}$. It only depends on the coupling strengths $J_{\perp}, J_{\|}$, and $J_{\text {imp }}$ via $J_{\mathrm{c}}$ [recall Eq. (3.13b)] and $\Delta \theta$ [recall Eq. (3.15b)]. In experiments, this ratio can be measured without knowing the density of impurity bonds [18].

\section{B. Dipolar approximation}

The mean-field theory of the preceding section has at least two drawbacks. As usual, the neglect of fluctuations will lead to an overestimate of the critical temperature by a certain factor $O(1)$, which might itself be a function of the ratios between the couplings. This makes it difficult to predict the precise dependence of $T_{\text {Ising }}$ on the couplings. A second and more serious drawback of these approximations is the fact that the site-averaged mean field of Eq. (6.4) receives rare but large contributions from pairs of sites that are nearest neighbors on the underlying lattice $\Lambda$. This contribution represents a nonvanishing fraction of the resulting mean field. However, physically it is clear that the Ising spins on very close pairs of sites will lock strongly together and either act as an effective spin with a doubled moment for ferromagnetic pairs or essentially decouple from the rest for antiferromagnetic coupling. In either case, these strong short-range couplings have essentially no influence on the long-range ordering, and thus it seems unphysical that such strong couplings should enter in our mean-field estimate of $T_{\text {Ising }}$ at all.

Here we follow a different approach to establish the dependence of $T_{\text {Ising }}$ on the couplings. Let $\xi$ be the length scale beyond which we can approximate the interactions $J_{\tilde{\boldsymbol{r}}-\tilde{\boldsymbol{r}}^{\prime}}^{(\mathrm{I})}$ as being antidipolar, i.e., given by Eq. (2.15). We assume that we can safely neglect pairs of Ising spins that are within a distance of order $\xi$ of each other [the probability to find another Ising spin a distance $\xi$ from a given one is of $O\left(\xi^{d} n_{\text {imp }}\right)$, a negligible probability as $n_{\mathrm{imp}} \rightarrow 0$ ]. If so, we may replace the Ising Hamiltonian (3.38) with the effective Ising Hamiltonian given by

$$
H_{\mathcal{L}}^{(\mathrm{eff})}\left[\sigma_{\tilde{\boldsymbol{r}}}\right]:=-\frac{1}{2} \sum_{\tilde{\boldsymbol{r}}, \tilde{\boldsymbol{r}}^{\prime} \in \mathcal{L}} \sigma_{\tilde{\boldsymbol{r}}} J_{\tilde{\boldsymbol{r}}-\tilde{\boldsymbol{r}}^{\prime}}^{(\mathrm{adip})} \sigma_{\tilde{r}^{\prime}}-\kappa n_{\mathrm{imp}} \frac{1}{|\mathcal{L}|} \sum_{\tilde{\boldsymbol{r}}, \tilde{\boldsymbol{r}}^{\prime} \in \mathcal{L}} \sigma_{\tilde{\boldsymbol{r}}} \sigma_{\tilde{r}^{\prime}}
$$

The parameter

$$
\kappa:=\frac{1}{2}\left(\Delta \theta \frac{J_{\mathrm{c}}+J_{\perp}}{J_{\perp}}\right)^{2} J_{\perp}
$$

determines the characteristic energy due to the coupling to the spiral twist (the infinite-range contribution to the Hamiltonian). The antidipolar interaction is

$$
J_{\tilde{\boldsymbol{r}}}^{\text {(adip) }}:=\frac{J_{0}}{2 \pi} \frac{\tilde{r}_{x}^{2}+\tilde{r}_{y}^{2}-2 \alpha \tilde{r}_{z}^{2}}{\left(\tilde{r}_{x}^{2}+\tilde{r}_{y}^{2}+\alpha \tilde{r}_{z}^{2}\right)^{5 / 2}},
$$

with the anisotropy of exchange couplings $\alpha \equiv J_{\|} / J_{\perp}$ [recall Eq. (3.21)] and the prefactor

$$
J_{0}:=\frac{1}{2} \sqrt{\frac{J_{\|}}{J_{\perp}}}\left(\Delta \theta \frac{J_{\mathrm{c}}+J_{\perp}}{J_{\perp}}\right)^{2} J_{\perp}=\sqrt{\alpha} \kappa .
$$

We expect that, for the purpose of determining the critical temperature, replacing Hamiltonian (3.38) with the Hamiltonian (6.11) is an excellent approximation.

We now claim that the critical temperature $T_{\text {Ising }}$ is well approximated by

$$
T_{\text {Ising }} \approx c \kappa n_{\mathrm{imp}}+O\left(n_{\mathrm{imp}}\right),
$$

with $c$ a number of $O(1)$, independent of $J_{\perp}, J_{\|}$, and $\Delta \theta$. Indeed, by assumption, $T_{\text {Ising }}$ is well approximated by the critical temperature of the Hamiltonian (6.11). Now we may trade the 
scaling transformation (2.13) for the scaling transformation

$$
\left(\begin{array}{c}
\tilde{r}_{x} \\
\tilde{r}_{y} \\
\sqrt{\alpha} \tilde{r}_{z}
\end{array}\right)=:\left(\frac{\sqrt{\alpha}}{n_{\mathrm{imp}}}\right)^{1 / 3}\left(\begin{array}{c}
\mathrm{r}_{x} \\
\mathrm{r}_{y} \\
\mathrm{r}_{z}
\end{array}\right),
$$

which preserves the Poissonian nature of the impurity distribution and is equivalent to replacing $J_{0}$ by $J_{0} n_{\mathrm{imp}} / \sqrt{\alpha}=\kappa n_{\mathrm{imp}}$ in Eq. (6.11). After this rescaling, we can factorize out the common energy scale $\kappa n_{\text {imp }}$ from both the dipolar and the spiral twist contributions to the Hamiltonian. The putative ordering temperature is then encoded in the dimensionless Hamiltonian

$$
\frac{1}{2} \sum_{\mathrm{r}, \mathrm{r}^{\prime} \in \mathcal{L}} \sigma_{\mathrm{r}}\left[-\frac{1}{2 \pi} \frac{\mathrm{r}_{x}^{2}+\mathrm{r}_{y}^{2}-2 \mathrm{r}_{z}^{2}}{\left(\mathrm{r}_{x}^{2}+\mathrm{r}_{y}^{2}+\mathrm{r}_{z}^{2}\right)^{5 / 2}}-\frac{2}{|\mathcal{L}|}\right] \sigma_{\mathrm{r}^{\prime}},
$$

which has a dimensionless ordering temperature $c=O(1)$ and in turn confirms our claim in Eq. (6.12). Numerical simulations of the antidipolar Ising model yield an estimate of the dimensionless prefactor to be $c \approx 1.5 .^{2}$

The above prediction for $T_{\text {Ising }}$ differs from the mean-field theory result $(6.5 \mathrm{a})$ by a factor of $\left(J_{\perp}+J_{\mathrm{c}}\right) / J_{\mathrm{c}}$ and additional numerical factors that in the case of mean-field theory might depend on the ratio of couplings. The deviation between the two approaches traces back to the various approximations made in the mean-field theory. From the result (6.12) we deduce that the ratio of the transition temperature to the spiral twist rate has the dependence

$$
\frac{T_{\text {Ising }}}{|Q|}=\frac{c}{2} \Delta \theta\left(J_{\mathrm{c}}+J_{\perp}\right)
$$

on the exchange couplings, where we recall that $\Delta \theta$ depends on all couplings $J_{\perp}, J_{\|}$, and $J_{\text {imp }}$ through the solution of Eq. (3.15b).

\section{Comparison to simulations in the $X Y$ model and to experiments}

We can now compare our theoretical predictions with experimental findings. Reference [13] reports a ratio $^{3} T_{\text {spi }} /|Q| \approx 60 \mathrm{meV}$ in $\mathrm{YBaCuFeO}_{5}$, while our theory predicts $T_{\text {Ising }} /|Q| \approx 68 \mathrm{meV}$ in the limit of low impurity density, upon using the couplings given in Eq. (4.12) (see Fig. 1). It is encouraging that our theory overestimates $T_{\text {spi }} /|Q|$ only by approximately $13 \%$, considering the simplifications that go into the modeling of the spin system

\footnotetext{
${ }^{2}$ We caution that rather large system sizes are necessary to reach the thermodynamic limit, as was already observed in Ref. [19], where the simulated system sizes for the full $X Y$ model were insufficient to reach the thermodynamic limit. Indeed, the apparent finite-size transition temperature exhibited a very significant size dependence. Here we have directly simulated the effective Ising model. While we reproduced the results of the full model for small samples, we were now able to reach much bigger sizes, where the transition temperature was found to saturate eventually, as expected. That saturation value was taken to estimate the value of $c$.

${ }^{3}$ References $[13,18]$ use a different convention for the spiral wave vector. Their wave vector $q_{G}$ is related to our $Q$ via the conversion $Q=\pi q_{G}$.
}

and the uncertainty in the value of the exchange couplings. As noted above, the ordering temperature is proportional to the concentration of impurity bonds. For the concrete case of $\mathrm{YBaCuFeO}_{5}$, our theory predicts that a small fraction of $1 \%$ of the oxygen bipyramids realizing the strongly frustrating Fe-Fe magnetic interactions induces a transition to the spiral phase at an ordering temperature of approximately $85 \mathrm{~K}$ (see Fig. 1). Note that at those temperatures the constraint of Eq. (3.16) is satisfied by a large margin and the mapping to the effective Ising model is thus well controlled.

A subsequent experimental study investigated a family of layered perovskites that all share the structural element of $\mathrm{Cu}$ and $\mathrm{Fe}$ hosting bipyramids with $\mathrm{YBaCuFeO}_{5}$ [18]. In those materials the lattice parameters were altered by chemical substitutions, which affect the exchange constants, especially the perpendicular coupling $J_{\perp}$. Alternatively, one can use uniaxial pressure to alter the lattice spacings. The concomitant changes to observables such as $T_{\text {spi }} /|Q|$ were recorded. The experiments of Ref. [18] show that the ratio $T_{\text {spi }} /|Q|$ is only very weakly sensitive to the modification of the interlayer spacing and thus of $J_{\perp}$.

These empirical findings can be rationalized by analyzing Eqs. (6.10) and (6.15). In layered perovskites, the exchange anisotropy between intra- and interlayer couplings is large. We model this empirical fact by requiring that $\alpha \equiv J_{\|} / J_{\perp} \gg 1$ [recall Eq. (3.21)]. Furthermore, the impurity coupling strength turns out to be large as well, $\left|J_{\text {imp }}\right| / J_{\|} \gg 1$ [recall Eq. (4.12)]. In this limit, $J_{\mathrm{c}} \approx 2 \pi J_{\|} /(\ln \alpha+2.47) \gg J_{\perp}$ [recall the approximation mentioned in the caption of Fig. 3]. The canting angle between the $X Y$ spins on either end of an impurity bond comes close to $\Delta \theta \approx \pi$ [recall Eq. (3.15b)]. More precisely, the deviation from $\pi$ is

$$
\pi-\Delta \theta \approx 2 \pi^{2} \frac{J_{\|}}{J_{\mathrm{imp}}\left[\ln \left(J_{\|} / J_{\perp}\right)+2.47\right]} .
$$

After dropping this correction, to a first approximation, the ratio between the critical temperature and the spiral twist rate at zero temperature can be approximated by

$$
\frac{T_{\text {Ising }}}{|Q|} \approx \frac{c \pi^{2} J_{\|}}{\ln \left(J_{\|} / J_{\perp}\right)+2.47},
$$

with the constant $c \approx 1.5$.

The degree to which the ratio $T_{\text {Ising }} /|Q|$ depends on the coupling $J_{\perp}$ can be quantified by the logarithmic derivative

$$
\frac{\partial \ln \left(T_{\text {Ising }} /|Q|\right)}{\partial \ln J_{\perp}} \approx \frac{1}{\ln \left(J_{\|} / J_{\perp}\right)+2.47} .
$$

For large anisotropy $\alpha$, this becomes small. For the experimental values of Eq. (4.12), the logarithmic derivative of Eq. (6.18) evaluates to approximately 0.2 , implying that a $50 \%$ change in $J_{\perp}$ only results in a $10 \%$ change of the ratio $T_{\text {Ising }} /|Q|$, in qualitative agreement with the experimental observations in Ref. [18].

\section{CONCLUSION AND OUTLOOK}

Any three-dimensional lattice hosting $X Y$ spins that interact through ferromagnetic nearest-neighbor exchange 
interactions display a ferromagnetic long-range order below some critical temperature. We have given sufficient conditions under which the replacement of a dilute fraction of the ferromagnetic bonds by antiferromagnetic bonds destabilizes the ferromagnetic order in favor of noncollinear long-range order in the form of a spiral phase. A necessary but not sufficient condition for spiral order is that the antiferromagnetic exchanges along the impurity bonds be sufficiently larger than the ferromagnetic couplings. This induces local canting, which lowers the energy close to the frustrating bond. If this condition is met, a sufficient condition for spiral order is a strong correlation between the impurity bonds such that (i) they all point along a preferred direction and (ii) they are distributed in space such that ferromagnetic interactions dominate between the Ising degrees of freedom associated with the local canting patterns around the impurities. We showed rigorously that (ii) is satisfied for impurities located on Bravais superlattices whose shortest lattice vectors tend to point in directions in which the effective Ising interactions are ferromagnetic, while neighboring impurities along the $z$ axis, for which the interactions are antiferromagnetic, appear only at larger distance. Small distortions away from a perfectly regular Bravais lattice (with displacements significantly smaller than the linear dimensions of a Voronoi unit cell of the superlattice) would in general not destroy the spiral order. Indeed, such small perturbations alter the effective interactions between the cantings only by a small fraction, which is not expected to destabilize the ferromagnetic ordering of cantings, if this ordering is a stable global minimum of the effective Ising model defined on the superlattice.

Superlattices do not arise very easily however. In superconducting analogs, which we will discuss below, superlattices of frustrated links could be nanofabricated. In magnetic materials superlattices of defects might form naturally if one changes the relative fraction of magnetic ions in perovskites (cf., for example, Ref. [36]) so as to induce a finite concentration of impurity bonds. Upon careful annealing, those can be expected to organize in spatially regular structures resembling a superlattice. However, since a change in ion composition may accompany a change in the valence of the ions on those bonds, it is not a priori clear whether they would still have a magnetically frustrating character.

So far, however, superlattices of impurity bonds have not been realized in actual materials. It is therefore an important result of the present work to show that completely randomly distributed impurities are always prone to stabilize spiral order, provided the impurity density is low enough. At higher impurity densities, a short-range repulsion among impurity bonds, e.g., due to Coulomb constraints in real materials, has the main effect of reducing the stability of fan states (layered antiferromagnetic orderings of the canting degrees of freedom), and thus also stabilizes spiral order. Hence, once the orientational correlation (i) is ensured, the tendency towards spiral order is rather strong. Below we review how property (i) arises in perovskite systems.

On the other hand, if the impurity bonds and their orientations are white-noise correlated in space, the microscopic $X Y$
Hamiltonian belongs to the family of three-dimensional random $X Y$ models introduced by Villain, which he predicted to generically host amorphous glassy order (dubbed a semi-spinglass). From this it follows that the zero-temperature phase diagram of three-dimensional random-bond $X Y$ magnets (as characterized by the strength of the frustrating antiferromagnetic interactions and their spatial correlations) contains at least four stable phases: the ferromagnetic phase, the spiral phase, the fan phase (i.e., ferromagnetic in plane order with orientation oscillating from plane to plane), and the semi-spinglass phase.

From the perspective of the original microscopic $X Y$ spins in the Hamiltonian (2.1), the phenomenology for small concentrations $n_{\text {imp }} \ll 1$ is the following. Upon lowering the temperature in the $X Y$ paramagnetic phase, a continuous phase transition takes place in the three-dimensional $X Y$ universality class to a ferromagnetic phase at the temperature $T_{X Y}$. This ferromagnetic phase becomes further unstable at the temperature $T_{\text {spi }} \ll T_{X Y}$ [as estimated by $T_{\text {Ising }}$ in Eq. (6.12)], where an $X Y$ spiral phase emerges via a continuous phase transition. It is driven by the dilute concentration $n_{\text {imp }} \ll 1$ of impurity bonds that are orientationally correlated. The spiral wave vector $Q$ may serve as an order parameter for this Ising transition. The associated critical exponents are expected to take mean-field values, given the dimensionality and the longrange nature of the dipolar interactions.

What happens as $n_{\mathrm{imp}}$ is increased so that $T_{\mathrm{spi}}^{\mathrm{MF}} \sim T_{X Y}$ ? In this limit, the effective Ising model (3.38) is no longer a valid approximation of Hamiltonian (2.1), so at this stage we cannot make controlled predictions. However, it seems very likely that at large enough $n_{\text {imp }} \lesssim 1$, the impurity bonds will dominate the coupling between adjacent $a b$ planes, inducing a layered antiferromagnetic state. Upon increasing $n_{\text {imp }}$ this state might be reached either continuously, with the spiral wave vector saturating at $Q=\pi$, or discontinuously, via a first-order transition at some critical value of $n_{\text {imp. In the }}$ regime of smaller $n_{\text {imp }} \lesssim 1$, the critical temperature of the ferromagnetic instability will decrease with increasing $n_{\text {imp }}$, while the spiral instability temperature is expected to continue to increase. They might merge into a single direct transition, if this is not preempted by the emergence of a layered antiferromagnetic phase. An approach that is nonperturbative in $n_{\text {imp }}$ is needed to address these questions. It remains an open challenge to determine optimal combinations of exchange couplings that would allow one to maximize $T_{\text {spi }}$ by increasing $n_{\text {imp }}$, and thereby extend the regime of the incommensurate spiral magnetic phase to high temperatures.

In our earlier paper [19] it was argued that $\mathrm{YBaCuFeO}_{5}$ and related layered perovskites unite all the essential ingredients of the Hamiltonian discussed in this work and thus could realize the spiral $X Y$ phase described above. Indeed, chemical disorder is believed to lead to occasional substitutions of $\mathrm{Fe}$ for $\mathrm{Cu}$ (and vice versa) in the bipyramids that are aligned along the $z$ axis. In the pristine material, those host a $\mathrm{Cu}-\mathrm{Fe}$ pair. By far the most pronounced effect of such substitutions is the fact that an impurity $\mathrm{Fe}-\mathrm{Fe}$ pair has a much stronger exchange of opposite sign as compared to a usual $\mathrm{Cu}-\mathrm{Fe}$ 
pair. The effect of such substitutions on the exchanges with neighboring magnetic ions is instead much smaller in comparison. These properties lead to the directional correlation (i) of impurity bonds, which are essential to stabilize a spiral phase. The supporting evidence is as follows. On the one hand, Monte Carlo simulations for realistic values of the magnetic exchange couplings in $\mathrm{YBaCuFeO}_{5}$ yield transition temperatures to the magnetic spiral phase as high as $250 \mathrm{~K}$. On the other hand, it was reported in Ref. [13] that tuning the degree of occupational disorder by changing the annealing procedure of $\mathrm{YBaCuFeO}_{5}$ affects the transition temperature and the wave vector of the spiral in a way that is qualitatively and quantitatively consistent with Eq. (6.15). Finally, we point out that a mechanism very similar to the one described here might be at work in hole-doped cuprates, where pairs of holes might take the role of the frustrating impurity bonds [37].

\section{Applications to other systems}

The main physical mechanism we discussed in this work applies to other systems as well. First, we point out that the restriction to $X Y$ spins is not essential. Indeed, we expect that Heisenberg spins with an $\mathrm{O}(3)$ symmetry (or any other set of continuous degrees of freedom undergoing spontaneous symmetry breaking) would exhibit essentially the same phenomenology: At low temperatures the unfrustrated system will order ferromagnetically. Frustrating antiferromagnetic impurity bonds induce local canting patterns that are subject to effective pairwise interactions upon integrating out spin waves. A ferromagnetic order of the canting degrees of freedom again implies spiral order for the original Heisenberg spins. If the canting induced by a local impurity bond preserves the coplanarity of the background ferromagnetic order, the problem simply reduces to an effective $X Y$ model. This is what we found to happen in the presence of nearest-neighbor Heisenberg interactions. However, with more complex interactions, it might occur that the local canting pattern is nonplanar. This would imply that the canting does not only have a discrete Ising degree of freedom, but rather a continuous $X Y$-like degree of freedom. Indeed, for an isolated impurity, any rotation of all spins around the direction of the background ferromagnetic magnetization yields an energetically equivalent canting pattern. Upon integrating out spin waves, these effective $X Y$ canting degrees of freedom will be coupled through dipolelike interactions and their ferromagnetic order will again induce a spiral of the original Heisenberg spins.

The phenomenology of magnetic $X Y$ spins immediately carries over to superconducting systems too. There the role of $X Y$ spins is taken by the phase of superconducting islands with a well established amplitude of the superconducting order parameter, and Josephson couplings replace the magnetic exchange couplings. Frustration could be induced by Josephson couplings with a negative sign (based on ferro- magnetic materials, for example). However, a much simpler way to achieve frustration consists in threading a homogenous magnetic flux through a Josephson junction array. The recent advances in fabrication techniques and nanolithography for such devices should allow one to artificially design and control $X Y$ systems with a desired spatial pattern of frustrated plaquettes that emulate the presence of the antiferromagnetic impurity bonds in the magnetic analog. A magnetic spiral phase with ferromagnetic order of the Ising degrees of freedom of the canting patterns then translates into a system of vortices of the same vorticity (sense of circulation), entailing a global supercurrent in the system. Exploration of this is left for future work.

\section{ACKNOWLEDGMENTS}

This research was partially supported by NCCR MARVEL, funded by the Swiss National Science Foundation. We would like to thank N. Spaldin, M. Troyer, M. Medarde, M. Kenzelmann, and M. Morin for useful discussions. We are grateful to M. Medarde for providing us with experimental data. H.S. acknowledges support from the DFG via FOR 1346, the SNF Grant No. 200021E-149122, ERC Advanced Grant SIMCOFE, and ERC Consolidator Grant CORRELMAT (Project No. 617196). This work was supported by JSPS KAKENHI Grants No. 16H01064 (J-Physics) and No. $16 \mathrm{~K} 17735$.

\section{APPENDIX: DEGENERACY OF ALL CONFIGURATIONS WITH FERROMAGNETIC ORDER IN THE PLANES}

Let us consider a maximally dense lattice of impurity bonds, i.e., $\mathcal{L}=\Lambda$, with $\Lambda$ the host cubic lattice. By comparing the interaction energies of various candidates for ground states we will establish that, in the dense limit, an infinite family of spin configurations is degenerate. These degenerate configurations are such that the Ising degrees of freedom take values that depend solely on the $z$ component of their position $\boldsymbol{r}=(x, y, z)$

$$
\sigma_{r} \equiv s_{z}= \pm 1 \text {. }
$$

Within any $x-y$ plane of the cubic lattice the Ising degrees of freedom are ferromagnetically ordered, but they are uncorrelated among different planes. According to Eq. (3.38), up to a global constant, the total energy per lattice site of such a configuration is

$$
\varepsilon\left[s_{z}\right]=-\frac{1}{2} \times \frac{1}{|\Lambda|} \sum_{\boldsymbol{r}^{\prime}, \boldsymbol{r}^{\prime \prime} \in \Lambda} J_{\boldsymbol{r}^{\prime}-\boldsymbol{r}^{\prime \prime}}^{(\mathrm{I})} s_{z^{\prime}} S_{z^{\prime \prime}} .
$$

We now focus on the interaction $E_{z^{\prime \prime} \mid z^{\prime}}$ between two layers with $z$ coordinates $z^{\prime}$ and $z^{\prime \prime}$, respectively. It is proportional to $s_{z^{\prime}} S_{z^{\prime \prime}}$, with

$$
-s_{z^{\prime}} S_{z^{\prime \prime}} E_{z^{\prime \prime} \mid z^{\prime}}:=\frac{1}{2} \sum_{x^{\prime}, y^{\prime}, x^{\prime \prime}, y^{\prime \prime}} J_{\boldsymbol{r}^{\prime}-\boldsymbol{r}^{\prime \prime}}^{(\mathrm{I})}=\sum_{x^{\prime}, y^{\prime}, x^{\prime \prime}, y^{\prime \prime}} \frac{\gamma}{2|\Lambda|} \sum_{\boldsymbol{k} \in \mathrm{BZ}(\Lambda) \backslash\{0\}} \hat{\Gamma}_{\boldsymbol{k}}^{(0)} e^{i \boldsymbol{k} \cdot\left(\boldsymbol{r}^{\prime}-\boldsymbol{r}^{\prime \prime}\right)}+\frac{\gamma L_{x} L_{y}}{2 J_{\perp} L_{z}},
$$


where $L_{x}, L_{y}$, and $L_{z}$ are the numbers of lattice sites along the $x, y$, and $z$ directions, respectively, and $|\Lambda|=L_{x} \times L_{y} \times L_{z}$. With the help of [recall Eq. (2.14b)]

$$
\hat{\Gamma}_{\left(k_{x}=0, k_{y}=0, k_{z} \neq 0\right)^{\top}}^{(0)}=\frac{1}{J_{\perp}},
$$

we can perform the sums over $x$ and $y$ coordinates. This sum gives

$$
-S_{z^{\prime}} S_{z^{\prime \prime}} E_{z^{\prime \prime}} \mid z^{\prime}=\frac{\gamma L_{x} L_{y}}{2 L_{z}} \sum_{\substack{k_{z} \\ k_{z} \boldsymbol{e}_{z} \in \mathrm{BZ}(\Lambda) \backslash\{\mathbf{0}\}}} \frac{e^{i k_{z}\left(z^{\prime}-z^{\prime \prime}\right)}}{J_{\perp}}+\frac{\gamma L_{x} L_{y}}{2 J_{\perp} L_{z}}=\frac{\gamma L_{x} L_{y}}{2 J_{\perp}} \delta_{z^{\prime}, z^{\prime \prime}} .
$$

The energy per spin in all configurations of arbitrarily layered, ferromagnetically ordered planes is thus $-\gamma / 2 J_{\perp}$, independently of the magnetization structure $s_{z}$.

[1] H. Katsura, N. Nagaosa, and A. V. Balatsky, Phys. Rev. Lett. 95, 057205 (2005).

[2] S.-W. Cheong and M. Mostovoy, Nat. Mater. 6, 13 (2007).

[3] D. Khomskii, Physics 2, 20 (2009).

[4] Y. Tokura and S. Seki, Adv. Mater. 22, 1554 (2010).

[5] M. Kenzelmann, A. B. Harris, S. Jonas, C. Broholm, J. Schefer, S. B. Kim, C. L. Zhang, S.-W. Cheong, O. P. Vajk, and J. W. Lynn, Phys. Rev. Lett. 95, 087206 (2005).

[6] T. Goto, T. Kimura, G. Lawes, A. P. Ramirez, and Y. Tokura, Phys. Rev. Lett. 92, 257201 (2004).

[7] M. Mochizuki and N. Furukawa, Phys. Rev. B 80, 134416 (2009).

[8] D. H. Lyons and T. A. Kaplan, Phys. Rev. 120, 1580 (1960).

[9] N. B. Ivanov, S. E. Krüger, and J. Richter, Phys. Rev. B 53, 2633 (1996).

[10] D. E. Cox, W. J. Takei, and G. Shirane, J. Phys. Chem. Solids 24, 405 (1963).

[11] S. Utsumi, D. Yoshiba, and N. Momozawa, J. Phys. Soc. Jpn. 76, 034704 (2007).

[12] M. Morin, A. Scaramucci, M. Bartkowiak, E. Pomjakushina, G. Deng, D. Sheptyakov, L. Keller, J. Rodriguez-Carvajal, N. A. Spaldin, M. Kenzelmann, K. Conder, and M. Medarde, Phys. Rev. B 91, 064408 (2015).

[13] M. Morin, E. Canévet, A. Raynaud, M. Bartkowiak, D. Sheptyakov, V. Ban, M. Kenzelmann, E. Pomjakushina, K. Conder, and M. Medarde, Nat. Commun. 7, 13758 (2016).

[14] B. Kundys, A. Maignan, and C. Simon, Appl. Phys. Lett. 94, 072506 (2009).

[15] V. Caignaert, I. Mirebeau, F. Bourée, N. Nguyen, A. Ducouret, J.-M. Greneche, and B. Raveau, J. Solid State Chem. 114, 24 (1995).

[16] Y. Kawamura, T. Kai, E. Satomi, Y. Yasui, Y. Kobayashi, M. Sato, and K. Kakurai, J. Phys. Soc. Jpn. 79, 073705 (2010).
[17] M. J. Ruiz-Aragón, E. Morán, U. Amador, J. L. Martínez, N. H. Andersen, and H. Ehrenberg, Phys. Rev. B 58, 6291 (1998).

[18] T. Shang, E. Canévet, M. Morin, D. Sheptyakov, M. T. Fernández-Díaz, E. Pomjakushina, and M. Medarde, Sci. Adv. 4, eaau6386 (2018).

[19] A. Scaramucci, H. Shinaoka, M. V. Mostovoy, M. Müller, C. Mudry, M. Troyer, and N. A. Spaldin, Phys. Rev. X 8, 011005 (2018).

[20] J. J. Alonso and J. F. Fernández, Phys. Rev. B 81, 064408 (2010).

[21] J. F. Fernández and J. J. Alonso, Phys. Rev. B 79, 214424 (2009).

[22] J. Villain, J. Phys. (Paris) 36, 581 (1975).

[23] J. Villain, J. Phys. C 10, 4793 (1977).

[24] J. Villain, J. Phys. C 11, 745 (1978).

[25] J. Villain, Z. Phys. B 33, 31 (1979).

[26] S. Dey, E. C. Andrade, and M. Vojta, Phys. Rev. B 101, 020411(R) (2020).

[27] P. Babkevich, N. Nikseresht, I. Kovacevic, J. O. Piatek, B. Dalla Piazza, C. Kraemer, K. W. Krämer, K. Prokeš, S. Mat'aš, J. Jensen, and H. M. Rønnow, Phys. Rev. B 94, 174443 (2016).

[28] J. J. Alonso, Phys. Rev. B 91, 094406 (2015).

[29] J. M. Luttinger and L. Tisza, Phys. Rev. 70, 954 (1946).

[30] A. I. Larkin and D. E. Khmel'nistskiı̌, Sov. Phys. JETP 29, 1123 (1969).

[31] R. B. Griffith, Phys. Rev. 176, 655 (1968)

[32] M. H. Cohen and F. Keffer, Phys. Rev. 99, 1128 (1955).

[33] A. Aharony and M. E. Fisher, Phys. Rev. B 8, 3323 (1973).

[34] A. Aharony, Phys. Rev. B 8, 3342 (1973).

[35] A. Aharony, Phys. Rev. B 8, 3349 (1973).

[36] A. I. Klyndyuk, E. A. Chizhova, and V. M. Kononovich, Russ. J. Inorg. Chem. 53, 1561 (2008).

[37] M. Capati, S. Caprara, C. Di Castro, M. Grilli, G. Seibold, and J. Lorenzana, Nat. Commun. 6, 7691 (2015). 